\title{
Methodology to estimate ionospheric scintillation risk maps and their contribution to position dilution of precision on the ground
}

\author{
Alexandra Koulouri $^{1}$ (D) Nathan D. Smith ${ }^{2} \cdot$ Bruno C. Vani $^{3} \cdot$ Ville Rimpiläinen $^{4} \cdot$ Ivan Astin ${ }^{5} \cdot$ Biagio Forte $^{5}$
}

Received: 27 March 2019 / Accepted: 6 January 2020 / Published online: 4 February 2020

(c) The Author(s) 2020

\begin{abstract}
Satellite-based communications, navigation systems and many scientific instruments rely on observations of trans-ionospheric signals. The quality of these signals can be deteriorated by ionospheric scintillation which can have detrimental effects on the mentioned applications. Therefore, monitoring of ionospheric scintillation and quantifying its effect on the ground are of significant interest. In this work, we develop a methodology which estimates the scintillation-induced ionospheric uncertainties in the sky and translates their impact to the end users on the ground. First, by using the risk concept from decision theory and by exploiting the intensity and duration of scintillation events (as measured by the $S_{4}$ index), we estimate ionospheric risk maps that could readily give an initial impression on the effects of scintillation on the satellite-receiver communication. However, to better understand the influence of scintillation on the positioning accuracy on the ground, we formulate a new weighted dilution of precision (WPDOP) measure that incorporates the ionospheric scintillation risks as weighting factors for the given satellite-receiver constellations. These weights depend implicitly on scintillation intensity and duration thresholds which can be specified by the end user based on the sensitivity of the application, for example. We demonstrate our methodology by using scintillation data from South America and produce ionospheric risk maps which illustrate broad scintillation activity, especially at the equatorial anomaly. Moreover, we construct ground maps of WPDOP over a grid of hypothetical receivers which reveal that ionospheric scintillation can also affect such regions of the continent that are not exactly under the observed ionospheric scintillation structures. Particularly, this is evident in cases when only the global positioning system is available.
\end{abstract}

Keywords Ionospheric scintillation · Risk · Dilution of precision · Statistics · Error covariances - Weights · South America · $S_{4}$ index $\cdot$ GNSS positioning accuracy $\cdot$ Scintillation monitor

\section{Introduction}

Many industrial and scientific applications exploit global navigation satellite system (GNSS) than includes global

Alexandra Koulouri

alexandra.koulouri@tuni.fi

1 Faculty of Information Technology and Communication Sciences, Tampere University, P.O. Box 692, 33101 Tampere, Finland

2 Bath, UK

3 Department of Informatics, Federal Institute of Education, Science and Technology of Sao Paulo, Campus Presidente Epitacio, Rua José Ramos Jr, 27-50 Presidente Epitacio, Sao Paulo, Brazil

4 Department of Physics, University of Bath, Bath BA2 7AY, UK

5 Department of Electronic and Electrical Engineering, University of Bath, Bath BA2 7AY, UK positioning system (GPS), globalnaya navigazionnaya sputnikovaya sistema, (GLONASS) and Galileo (European navigation system) for precision positioning, agriculture, transportation, surveying, construction and geodesy among others. Satellite links using frequencies up to a few $\mathrm{GHz}$ (VHF through L-band) can experience significant signal amplitude and phase fluctuations due to small-scale plasma density inhomogeneities, a phenomenon known as scintillation (Crane 1977; Aarons 1982). The amplitude scintillation (i.e. temporal fluctuation of the signal strength) and phase scintillation occur often at lower latitudes and can cause periods of reduced signal power at the receiver's antenna (fading). Therefore, GNSS signal-to-noise ratio may drop below the receiver-tracking threshold (Jiao and Morton 2015), thereby leading to loss of positioning information, that can persist for a significant period of time (Aquino et al. 2005).

Many characteristics of scintillation are well studied (Aarons 1982; Basu et al. 1988; Conker et al. 2003; Li et al. 
2010; Béniguel et al. 2011; Jiao and Morton 2015; Forte et al. 2017; Sahithi et al. 2019). It has been shown that scintillation activity varies with operating frequency, local time, season, geomagnetic activity level (planetary $K$-index, $K_{p}$ ), and the 11-year solar cycle (Skone 2001; Li et al. 2008, 2010; Olatunbosun 2017; Basu et al. 1988; Jiao and Morton 2015). Moreover, the scintillation effects vary according to the user's location. During high-scintillation activity, these effects can be a serious problem for GNSS users in certain regions, such as polar and equatorial latitudes, while users in other regions (e.g. middle latitudes) are not affected to a large extent (Spogli et al. 2009; Abadi et al. 2014; Jiao and Morton 2015; Luo et al. 2018). Equatorial regions experience strong scintillation mostly after sunset until a few hours after midnight local time (Paul and DasGupta 2010; Jiao and Morton 2015; Jiao et al. 2016). In the equatorial ionosphere, the most significant scintillation activity occurs with deep signal fades causing a GNSS receiver to lose lock and degrade positioning accuracy (Skone et al. 2001; Aquino et al. 2005; Moreno et al. 2011; Marques et al. 2015).

Limited case studies have been carried out related to the effects of scintillation for the end user (Zumberge et al. 1997; Moreno et al. 2011; Sreeja et al. 2011; Xu et al. 2012; Pi et al. 2017; Alfonsi et al. 2018; Luo et al. 2018; de Moraes et al. 2018; Guo et al. 2019), and often global climatological models fail to describe extreme day-to-day variability of scintillation (Wernik et al. 2003). Moreover, because scintillation is frequency dependent and cannot be eliminated, for example, by combining observable signals at different frequencies (Mainul and Jakowski 2012; van den IJssel et al. 2016), the constant monitoring of scintillation activity using in situ measurements alongside with the development of mathematical methodologies, which describe, quantify and interpret its impact on GNSS communications, is substantial to mitigate its effect. In this framework, we develop a mathematical methodology to estimate ionospheric scintillation risks based on scintillation measurements and decision theory. These risks are given by the joint probability of scintillation intensity and duration estimated using information obtained from ground-based scintillation monitors. Thus, in contrast to the commonly estimated statistics (e.g. averages or percentages of scintillation measurements above a threshold as in (Spogli et al. 2009; Li et al. 2010; Sreeja et al. 2011; Jacobsen and Dähnn 2014), we use data collected from a network of scintillation monitors to construct ionospheric risk maps that describe and visualize scintillation behaviour. While the ionospheric maps can readily be used to get an (initial) impression of the effects of scintillation on a satellite-receiver communication, in this work, we propose to incorporate the scintillation risk into the receiver's position dilution of precision (PDOP) (Langley 1999).

Standard PDOP is a well-known way to quantify the reliability and integrity of a GNSS positioning system by using the geometry of the available satellite constellations (Sairo et al. 2003; Zhang et al. 2013; Chen et al. 2013; Tahsin et al. 2015; Teng et al. 2018). Here, we show how the degradation of PDOP occurs relative to the location of ionization structures in the F-region due to scintillation. To do that, we propose to use a weighted position dilution of precision (WPDOP) which is estimated by assigning different weights to receiver-satellite links using the ionospheric scintillation risks along the corresponding line-of-sights. Hence, in addition to the number and the geometry of the visible satellites, we take into account the effect of scintillation. Previous works have employed empirical error tracking models, e.g. Conker model (Conker et al. 2003), to implicitly model underlying scintillation activity by weighting receiver's measurements with the inverse of the variance of the tracking error. However, these approaches are often susceptible to model limitations and requirements of heuristically selected parameters and their applicability is limited due to the requirement of specific software/hardware that is available mainly in operating ground stations and not in standard GNSS receivers (Aquino et al. 2009; da Silva et al. 2010). Instead, our approach utilizes weighing factors based on risks associated directly with monitored scintillation data. With the help of these weights, we are able to construct maps that describe the expected uncertainty in positioning results during ionospheric scintillation. To the best of our knowledge, this is the first time such a methodology is introduced.

The rest of the paper is organized as follows. In Sect. 2, we present the theoretical background and mathematical expression of the risks and the proposed WPDOP. In Sect. 3, we describe the formulae and technical details for the implementation of the proposed ionospheric risks and the corresponding ground WPDOP values. In Sect. 4, we demonstrate our methodology by estimating ionospheric risk maps over South America using real scintillation data. Subsequently, we construct ground maps based on two different setups using either only GPS or GPS, GLONASS and Galileo. Then, we discuss the main observations and further potentials of the proposed methodology. Finally, in Sect. 5, we present a summary of our work and future aims.

\section{Methodology}

\subsection{Risk concept}

While it may be straightforward to extract statistics (e.g. averages or standard deviations) to describe scintillation (Liu et al. 2015), nevertheless it is important to interpret those statistics for an end user, in terms of the costs expected for a particular application when scintillation is experienced. For this purpose, we use risk analysis from decision theory (Oppe 1988; Murphy 2012). Risk can be defined as the expected 
loss incurred when an activity is performed (for example, satellite-receiver communication). To estimate the risk, we need (i) to find features that describe the condition in which we perform this activity (e.g. communication under scintillation can be described through the measured $S_{4}$ value), (ii) the frequency of occurrence of these features and (iii) how injurious the condition described by these features is to the successful outcome of the activity. These are, respectively, expressed by (i) a feature vector, (ii) a probability and (iii) a loss function.

In this section, we describe the background theory and definitions about loss function and risk before making it application-specific in the next section. Here, we denote random variables by capital letters and their realizations by lowercase letters. We start with the measurable feature $Z \in \mathscr{Z}$, where $\mathscr{Z}$ represents the set of all possible feature vectors (e.g. scintillation index, etc).

Let us introduce a $0-1$ loss function

$\ell\left(z ; z_{\text {th }}\right)= \begin{cases}0 & \text { if } z<z_{\text {th }} \\ 1 & \text { otherwise }\end{cases}$

which implies there is no loss when values of $z$ are less than a specified threshold, but otherwise losses are equally injurious. Although this is a simple loss function, it nevertheless is instructive for this particular problem without increasing computational complexity, and as will be shown it permits a straightforward interpretation.

The risk is then defined as the expected value of the loss function given by

$r\left(z_{\text {th }}\right)=\mathbb{E}\left[\ell\left(z ; z_{\text {th }}\right)\right]=\int_{\mathscr{Z}} \ell\left(z ; z_{\text {th }}\right) p(z) \mathrm{d} z$

where $\mathbb{E}[$.$] denotes the expectation value and p(z)$ is the probability density function of the measurable feature $Z$. From Eqs. 1 and 2, we have that

$r\left(z_{\text {th }}\right)=\pi\left(Z \geq z_{\text {th }}\right)$.

where $\pi($.$) denotes probability.$

Thus, the risk has a simple interpretation in terms of probability.

\subsection{Risk associated with ionospheric scintillation}

In this section, we apply the previous analysis to estimate risk maps using scintillation features. Let $X$ denote a scintillation index (e.g. $S_{4}$ value). Moreover, we define as a scintillation event a time series of scintillation index values above a scintillation threshold denoted by $x_{\text {th }}$ over a duration limit $d_{\text {th }}$ (see Fig. 1). The duration of scintillation events can be treated as

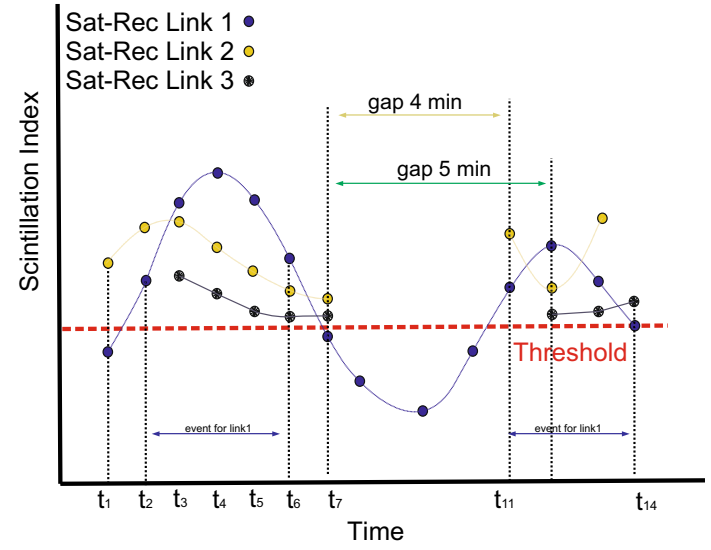

Fig. 1 A schematic example which shows how a scintillation event is determined. This figure shows IPP data samples (depicted with circles) in one ionospheric pixel over a short interval of time (the sampling period is considered $1 \mathrm{~min}$ ). Each colour corresponds to a data sequence from a different satellite-ground station link. IPP values greater than the intensity threshold (depicted by the horizontal dashed line) indicate that the sample belongs to a scintillation event. For link 1, we can observe that there are two distinct scintillation events between $\left[t_{2}-t_{6}\right]$ and $\left[t_{11}-\right.$ $\left.t_{14}\right]$. Links 2 and 3 have data gaps of duration 4 and 5 min, respectively. If the gap threshold is $4 \mathrm{~min}$, then we can consider a single scintillation event for link 2 and two scintillation events of short durations for Link 3

a random variable denoted by $D$. Based on the analysis of Sect. 2.1, we have that $(X, D) \equiv Z$.

Using the insight that the vulnerability of a link depends on the value of the scintillation index (e.g. strong scintillation when $S_{4}>0.6$ ) and duration of a scintillation event, we define the loss function

$\ell\left(x, d ; x_{\mathrm{th}}, d_{\mathrm{th}}\right)= \begin{cases}0 & \text { if } x<x_{\mathrm{th}} \text { and } d<d_{\mathrm{th}} \\ 1 & \text { otherwise }\end{cases}$

where $x_{\text {th }}$ and $d_{\text {th }}$ are the intensity and duration thresholds, respectively. These thresholds can be chosen to reflect the specifications of the receiver and the needs of safety critical applications.

From Eq. 3, the risk due to scintillation features is

$r_{\text {ion }}\left(x_{\mathrm{th}}, d_{\mathrm{th}}\right)=\pi\left(X \geq x_{\mathrm{th}}, D \geq d_{\mathrm{th}}\right)$.

Hence, the risk of the underlying activity depends on the joint probability of the duration and intensity of scintillation events and takes values between 0 and 1 .

\subsection{Effect of scintillation on the positioning accuracy}

The proposed risk can readily be used to give an initial impression of the effect of scintillation in a satellite-receiver communication. However, to better understand the influence of scintillation on the positioning accuracy on the ground, 
a well-known quantity as the dilution of precision can be estimated (Langley 1999).

Towards this aim, this section introduces a weighted position dilution of precision (WPDOP) as a measure of uncertainty in estimating the receiver's position. To put our analysis in perspective, we first discuss the theoretical basis of the standard position dilution of precision (PDOP) from the Bayesian point of view. Then, we explain how in addition to the availability and geometry of the satellites, the proposed WPDOP can include information about the effect of ionospheric scintillation.

\subsubsection{Standard position dilution of precision}

Following similar analysis as in (Langley 1999), for a ground receiver at location $r=\left(r_{x}, r_{y}, r_{z}\right)^{\mathrm{T}}$, the positioning error at time $t$, denoted by $\Delta r=\left(\Delta r_{x}, \Delta r_{y}, \Delta r_{z}\right)^{\mathrm{T}}$, can be expressed through the linear system

$b=A \Delta r+\varepsilon$,

where $b \in \mathbb{R}^{S}$ is a vector with the differences between the measured and modelled pseudorange values, $A=$ $\left[\mathrm{n}_{1}, \ldots, \mathrm{n}_{S}\right]^{\mathrm{T}} \in \mathbb{R}^{S \times 3}$ where $\mathrm{n}_{S}$ is a unit column vector pointing from the modelled (approximated) receiver position to the satellite and $S$ is the total number of visible satellites at a time instance $t$. Error $\varepsilon \in \mathbb{R}^{S}$ represents the measurement noise plus model errors and ionospheric effects (including scintillation). Further details about the derivation of the aforementioned linear system are given in "Appendix A". 1

In the PDOP standard probabilistic analysis (Langley 1999; Misra and Enge 2010), the noise $\varepsilon$ is modelled as i.i.d. Gaussian given by $\varepsilon \sim \mathscr{N}\left(0, \gamma I^{S \times S}\right)$ (with constant error variance $\gamma>0$ and $I S \times S$ identity matrix). A point estimate for the positioning error $\Delta r$ can be obtained by solving

$\widehat{\Delta r}:=\arg \max _{\Delta r} p(\Delta r \mid b)$,

where $p(\Delta r \mid b)$ is the posterior distribution of $\Delta r$ given by $p(\Delta r \mid b) \propto p(b \mid \Delta r) p(\Delta r)$ based on the Bayes theorem, where $p(b \mid \Delta r) \propto \exp \left\{-\frac{1}{2 \gamma}(A \Delta r-b)^{\mathrm{T}}(A \Delta r-b)\right\}$ is the likelihood and $p(\Delta r)$ is the prior distribution (Kaipio and Somersalo 2004).

With a non-informative prior $p(\Delta r)$, the positioning error can be obtained by $\widehat{\Delta r}:=\arg \max _{\Delta r} p(b \mid \Delta r)$ which is the maximum likelihood (ML) estimate. The ML estimate is

\footnotetext{
1 We note here that the clock offset is omitted (as a variable to be estimated) from the current formulation. Matrix $A, \Delta r, b$ and $\varepsilon$ are time-varying variables, but for simplicity in the notation, time $t$ has not been used in this context. Also, linear system (Eq. 6) helps us to introduce the different dilution of precision formulae. The estimation of vector $b$ is out of the scope of the current work, the interested reader is referred to, for example, Teunissen and Kleusberg (1998).
}

$\widehat{\Delta r}=\left(A^{\mathrm{T}} A\right)^{-1} A^{\mathrm{T}} b$,

and it is equal to the least squares solution of system (Eq. 6). The solution (Eq. 8) exists as long as there are 3 or more satellites located in distinct directions in the sky. Quantitatively, the rank of $\left(A^{\mathrm{T}} A\right)$ can be used as an indicator whether the problem is well posed or not. For example, if $\operatorname{rank}\left(A^{\mathrm{T}} A\right) \geq 3$, then we can deduce that there are enough satellites for the position estimation. However, the confidence in the solution (Eq. 8) depends on the inverse matrix $\left(A^{\mathrm{T}} A\right)^{-1}$ (whether it is well or ill conditioned). From the Bayesian point of view, this inverse matrix multiplied by error variance $\gamma$ is the posterior covariance of $p(\Delta r \mid b)$ given by $\Gamma=\gamma\left(A^{\mathrm{T}} A\right)^{-1}$ and its diagonal elements (posterior variances) can help to quantify the confidence in the estimate $\widehat{\Delta r}$ (Kaipio and Somersalo 2004). Large posterior variances may indicate that there is possibly an overlapping between satellites (eclipse phenomenon), collinearity or inappropriate geometric constellation. The square root of the trace of the posterior covariance $\Gamma$ normalized by the constant variance $\gamma$ is the well-known position dilution of precision (PDOP) (Langley 1999). In particular, for a ground receiver at location $r$ and time $t$, the PDOP is given by

$\mathrm{PDOP}^{r, t}=\sqrt{\frac{\operatorname{tr}(\Gamma)}{\gamma}}=\sqrt{\operatorname{tr}\left(\left(A^{\mathrm{T}} A\right)^{-1}\right)}$,

where $\operatorname{tr}()$ denotes the trace of matrix given by $\operatorname{tr}\left(\left(A^{\mathrm{T}} A\right)^{-1}\right)$ $=\sum_{s=1}^{S} \operatorname{diag}\left(\left(A^{\mathrm{T}} A\right)^{-1}\right)$.

\subsubsection{Weighted position dilution of precision}

Now, by considering the scintillation effect independently from all the other errors, the noise term in the observation model (Eq. 6) can be decomposed into two uncorrelated errors

$\varepsilon=\varepsilon_{\mathrm{sc}}+\varepsilon_{\text {rem }} \in \mathbb{R}^{S}$.

The error due to ionospheric scintillation can be approximated by a Gaussian distribution given by $\varepsilon_{\mathrm{sc}} \sim \mathscr{N}\left(0, \Gamma_{\varepsilon_{\mathrm{sc}}}\right)$ (Misra and Enge 2010) where its covariance is a diagonal matrix $\Gamma_{\varepsilon_{\mathrm{sc}}}=\operatorname{diag}\left(\gamma_{\mathrm{sc}}^{(s)}\right)$ with the variances depending on the scintillation risks along the available satellite-receiver ray-paths. Therefore, the error variances due to scintillation can be expressed as a function of the ionospheric risk $r_{\text {ion }}^{(s)}$ along each ray-path $s$, i.e.

$\gamma_{\mathrm{sc}}^{(s)}=h\left(r_{\text {ion }}^{(s)}\right)$ for $s=1, \ldots, S$

Function $h\left(r_{\text {ion }}^{(s)}\right)$, since it is the variance of the measurement errors caused by scintillation in link $s$, should be a 
positive monotonically non-decreasing function and satisfy $h\left(r_{\text {ion }}^{(s)}\right) \rightarrow \infty$ for risk $r_{\text {ion }}^{(s)} \rightarrow 1$ (more uncertainty is introduced in the estimates due to scintillation) and $h\left(r_{\text {ion }}^{(s)}\right) \rightarrow 0$ when risk $r_{\text {ion }}^{(s)} \rightarrow 0$ (minimal effects due to scintillation in the estimates when risk is low).

The remaining error $\varepsilon_{\text {rem }}$, that encloses all the other modelling and measurement related uncertainties, is modelled as Gaussian, i.e. $\varepsilon_{\text {rem }} \sim \mathscr{N}\left(0, \gamma I^{S \times S}\right)$ with $\gamma$ constant as in the standard PDOP analysis ${ }^{2}$ (Langley 1999). We notice that in the current analysis the errors between different links are considered uncorrelated which is a plausible assumption since there are usually large distances between different raypaths. $^{3}$

Then, the covariance matrix of total error $\varepsilon$ given by $\Gamma_{\varepsilon}=$ $\gamma I^{S \times S}+\Gamma_{\varepsilon_{\mathrm{sc}}}$ is a diagonal matrix $\Gamma_{\varepsilon}=\operatorname{diag}\left(\gamma_{\varepsilon^{(s)}}\right)$ with elements

$\gamma_{\varepsilon^{(s)}}=\gamma+h\left(r_{\text {ion }}^{(s)}\right)$ for $s=1, \ldots, S$.

The positioning error estimate is now

$\widehat{\Delta r}=\left(A^{\mathrm{T}} \Gamma_{\varepsilon}^{-1} A\right)^{-1} A^{\mathrm{T}} \Gamma_{\varepsilon}^{-1} b$,

when $S \geq 3$ and $\operatorname{rank}\left(A^{\mathrm{T}} \Gamma_{\varepsilon}^{-1} A\right) \geq 3$. The posterior covariance of $\Delta r$ is

$\Gamma=\left(A^{\mathrm{T}} \Gamma_{\varepsilon}^{-1} A\right)^{-1}$

and the weighted position dilution of precision (WPDOP) for a ground receiver at location $r$ and time $t$ is defined (in a similar way as in Eq. 9) as the square root of the trace of the posterior $\Gamma$ normalized by the constant $\gamma$ and it is given by

$$
\begin{aligned}
\mathrm{WPDOP}^{r, t} & =\sqrt{\frac{\operatorname{tr}(\Gamma)}{\gamma}} \\
& =\sqrt{\operatorname{tr}\left(A^{\mathrm{T}}\left(\frac{\Gamma_{\varepsilon}}{\gamma}\right)^{-1} A\right)^{-1}}=\sqrt{\left(\operatorname{tr}\left(A^{\mathrm{T}} W A\right)^{-1}\right)}
\end{aligned}
$$

\footnotetext{
2 Gaussian distribution with constant variance is a standard modelling assumption when prior statistical information about the observation error is limited or unavailable (Misra and Enge 2010).

${ }^{3}$ For GPS L1 frequency, the most effective spatial size of the electron density irregularities that causes amplitude scintillation is of order 400 m (first Fresnel scale at $\sim 350 \mathrm{~km}$ altitude) (Rufenach 1972; Kintner and Ledvina 2005; Peng and Scales 2019). The IPPs of two different ray-paths are separated by distances that are much larger than the size of such a single scintillation structure. Therefore, it is very unlikely for two ray-paths to cross the same ionospheric structure.
}

where $\operatorname{tr}\left(\left(A^{\mathrm{T}} W A\right)^{-1}\right)=\sum_{s=1}^{S} \operatorname{diag}\left(\left(A^{\mathrm{T}} W A\right)^{-1}\right)$ and $W=\left(\frac{\Gamma_{\varepsilon}}{\gamma}\right)^{-1}$ is a diagonal matrix, with elements

$w_{s}=\frac{\gamma}{\gamma_{\varepsilon}^{(s)}}=\frac{\gamma}{\gamma+h\left(r_{\text {ion }}^{(s)}\right)}$ for $s=1: S$,

where $0 \leq w_{s} \leq 1$ and $\gamma$ constant. A rigorous derivation of Eq. 15 is given in "Appendix B".

Based on Eq. 16, we have that $w_{s}=1$ when $r_{\text {ion }}^{(s)}=0$ (no scintillation, since $\left.h\left(r_{\text {ion }}^{(s)}\right)=0\right)$, and $w_{s}=0$ when $r_{\text {ion }}^{(s)} \rightarrow 1$ (if $h\left(r_{\text {ion }}^{(s)}\right) \rightarrow \infty$ ). The shape of $h($ ) in Eq. 16 may be selected based on prior knowledge or application requirements. In general, the proposed WPDOP uses both information about the number of available satellites, their geometry in the sky and the risks associated with scintillation along the visible ray-paths. If $\operatorname{rank}\left(A^{\mathrm{T}} W A\right)<3$, i.e. the number of available satellites is less than 3 , then $\mathrm{WPDOP}^{r, t}$ is undefined.

\section{Implementation}

\subsection{Single shell ionospheric model}

The F-region (especially around $350 \mathrm{~km}$ of altitude) is of main concern for GNSS users due to propagation errors caused by the dynamics of the large electron density. To construct surface ionospheric maps, it is standard to consider the ionospheric thin shell approximation at $350-\mathrm{km}$ altitude, based on the assumption that the entirety of the electron content (which affects a link) is acting at the point where a ray-path of a satellite-receiver intersects (or pierces) the ionospheric shell at that altitude (Davies 1990). Hence, an ionospheric risk map can be estimated using ground measurements that are directly projected on the ionospheric shell at the corresponding ionospheric pierce points (IPPs) ${ }^{4}$ (similarly as in (Vani et al. 2017)). The projected (scintillation) measurements at the $350-\mathrm{km}$ ionospheric shell will be referred to as IPP data in the following text.

\section{2 lonospheric computational domain and data accumulation}

For the construction of an ionospheric risk map, the thin ionospheric shell was discretized using a uniform grid where each pixel got its own risk value. In Sect. 4.5, we estimated scintillation risk maps over South America and as a reliable index of scintillation at low latitudes, we used $S_{4}$ data which are the ratio of the standard deviation of the received signal intensity (measured by a $50-\mathrm{Hz}$ sampling rate) to the averaged

\footnotetext{
${ }^{4}$ IPP is referred to as the point where a line-of-sight from the ground receiver to a satellite "punctures" the ionosphere at the ionospheric shell.
} 
intensity during an 1-min time interval. A risk was estimated at each ionospheric pixel $v$ based on the joint probability (Eq. 5) using IPP data accumulated over a time interval $\tau$. We note that there is an implicit assumption of statistical stationarity, i.e. the joint probability does not change over time.

\subsection{A scintillation event}

A scintillation event was introduced briefly in Sect. 2. Here, we explain more analytically the properties of such events. Particularly, duration of a scintillation event is the time interval where the scintillation values for a specific (satellitescintillation monitor) link are greater than a given threshold. In the schematic example of Fig. 1, we show how different scintillation events are determined using sequences of IPP data samples (depicted by small circles) for 3 different links imagined that their ray-paths are passing through the same ionospheric pixel $v$. The horizontal dashed line indicates a selected intensity threshold $x_{\text {th }}$. All the links experience scintillation events since there are data points above the threshold. For link 1 (in blue), we can observe that there are two distinctive scintillation events between $\left[t_{2}-t_{6}\right]$ and $\left[t_{11}-t_{14}\right]$. However, for links 2 (in yellow) and 3 (in black) we have to deal with data gaps related to either strong scintillation or other reasons. In these cases, we have to decide whether the available data and the corresponding gaps are considered as a single scintillation event or not. In the current implementation, we consider that there is a single scintillation event if the gap is small (i.e. less than $4 \mathrm{~min}$ ). For a longer gap, we consider that two separate scintillation events of shorter durations take place. Extra conditions based on the values of the scintillation samples before and after the gap may also be applied to differentiate single or multiple events. For example, very high values of the $S_{4}$ samples on either side of a large gap (e.g. $S_{4} \geq 0.6$ ) can imply a single event rather than two distinct events.

\subsection{Risk estimation}

In this section, we describe the mathematical formulae for the estimation of the ionospheric risk maps. As we mentioned earlier, risk maps are constructed by estimating pixelwise the joint probability (Eq. 5) using the accumulated IPP data at each pixel of the discretized ionospheric domain over a period of time $\tau$. Based on that and Eq. 5, we can mathematically express a risk in a pixel as the conditional probability given pixel $v$ and time window $\tau$, i.e.

$r_{\text {ion }}^{v, \tau}\left(x_{\mathrm{th}}, d_{\mathrm{th}}\right)=\pi\left(X \geq x_{\mathrm{th}}, D \geq d_{\mathrm{th}} \mid v, \tau\right)$.

Moreover, we introduce the following notation for clarity. A scintillation measurement is given by $x^{s}(\mathrm{v}(t))$, where $\mathrm{v}(t)=$ $\left(v_{x}(t), v_{y}(t)\right)$ is the geographic location (at the ionospheric pierce point) at time $t$. Superscript $s$ is used to identify that the measured index corresponds to a particular satellite-monitor link. Then, a scintillation event is a time series of scintillation samples $x^{s}(\mathrm{v}(t)) \geq x_{\text {th }}$ for an interval $[t, t+\Delta d]$, where $d \geq d_{\text {th }}$ and when these belong to a single pixel, $v$ (i.e. $\mathrm{v}(t) \in v)$. Here, $\Delta$ is the sampling period and $d_{\text {th }}$ is an integer denoting the minimum number of samples required to qualify for the event (a.k.a. duration threshold). Thus, each IPP sample can be represented pairwise as $\left(x^{s}(\mathrm{v}(t)), d^{s}(t)\right)$ that includes the scintillation value and the number of samples in the scintillation event to which $x^{s}(\mathrm{v}(t))$ belongs. For example, $d^{s}(t)=0$ when $x^{s}(\mathrm{v}(t))<x_{\text {th }}$ which means that sample $x^{s}(\mathrm{v}(t))$ does not belong to a scintillation event. On the other hand, when $x^{s}(\mathrm{v}(t)) \geq x_{\text {th }}$ then sample $x^{s}(\mathrm{v}(t))$ belongs to a scintillation event and then $d^{s}(t)$ equals the number of consecutive samples (before and after (and including) $x^{s}(\mathrm{v}(t))$ at time $t$ ) that exceed threshold $x_{\text {th }}$. In the following analysis, for simplicity in the probability expressions, we write measurement $\left.x^{s}(\mathrm{v}(t))\right|_{t=t_{j}}=x^{s}[j]$, where $x^{s}[j]$ is the value of the scintillation index at time $t_{j}$ for satellite-receiver link $s$ and the corresponding IPP (ionospheric location) of $x^{s}[j]$ is denoted by $\mathrm{v}^{s}[j]$.

The joint probability of $X$ and $D$ (Eq. 17) can be re-written as

$$
\begin{gathered}
\pi\left(X \geq x_{\mathrm{th}}, D \geq d_{\mathrm{th}} \mid v, \tau\right)=\pi\left(D \geq d_{\mathrm{th}} \mid X \geq x_{\mathrm{th}}, v, \tau\right) \\
\pi\left(X \geq x_{\mathrm{th}} \mid v, \tau\right) .
\end{gathered}
$$

The previous equation can be further expanded, i.e.

$$
\begin{array}{r}
\pi\left(X \geq x_{\mathrm{th}}, D \geq d_{\mathrm{th}} \mid v, \tau\right)=\sum_{d_{k} \geq d_{\mathrm{th}}} \\
\pi\left(D=d_{k} \mid X \geq x_{\mathrm{th}}, v, \tau\right) \pi\left(X \geq x_{\mathrm{th}} \mid v, \tau\right),
\end{array}
$$

where $d_{k}$ is an integer denoting the number of samples $\left(x^{s}[j], \ldots, x^{s}\left[j+d_{k}\right]\right) \geq x_{\text {th }}, x^{s}[j-1]<x_{\text {th }}$ and $x^{s}\left[j+d_{k}+1\right]<x_{\mathrm{th}}$, which corresponds to a temporal duration of $d_{k} \Delta$.

Based on Eq. 19, risk (Eq. 17) is estimated by first calculating probability $\pi\left(X \geq x_{\mathrm{th}} \mid v, \tau\right)$ according to

$\pi\left(X \geq x_{\mathrm{th}} \mid v, \tau\right)=\frac{\sum_{s=1}^{S} \sum_{j=0}^{N_{s}-1} U\left(x^{s}[j] \mid v, \tau, x_{\mathrm{th}}\right)}{\sum_{s=1}^{S} \sum_{j=0}^{N_{s}-1} U\left(x^{s}[j] \mid v, \tau, 0\right)}$,

where $U$ is a step function defined as

$$
\begin{aligned}
& U\left(x^{s}[j] \mid v, \tau, \alpha\right) \\
& \quad= \begin{cases}1 & \text { if } x^{s}[j] \geq \alpha \text { given that } \mathrm{v}^{s}[j] \in v \text { and } t_{j} \in \tau \\
0 & \text { otherwise },\end{cases}
\end{aligned}
$$


index $S$ is the total number of available links, $\alpha$ is the intensity threshold, and $N_{S}$ the number of the available scintillation samples (i.e. total number of measurements) for link $s$ over a time window $\tau$.

Furthermore, the conditional probability $\pi\left(D=d_{k} \mid X \geq x_{\mathrm{th}}, v, \tau\right)$ is

$$
\begin{aligned}
\pi(D & \left.=d_{k} \mid X \geq x_{\mathrm{th}}, v, \tau\right) \\
& =\frac{\sum_{s=1}^{S} \sum_{j=0}^{N_{s}-1} H_{d}\left(x^{\tau}[j], \ldots, x^{s}\left[j+d_{k}\right] \mid v, \tau, x_{\mathrm{th}}\right) d_{k}}{\left.\sum_{s=1}^{S} \sum_{j=0}^{N_{s}-1} U\left(x^{s}[j] \mid v, \tau, x_{\mathrm{th}}\right)\right)},
\end{aligned}
$$

spheric risk map at $350 \mathrm{~km}$. Weight (Eq. 25) satisfies the limiting values for $w_{s}$ (Eq. 16) as risk varies and parameter $k \geq 0$ penalizes our trust to a ray-path based on the scintillation risk value. For the estimation of WPDOP in Sect. 4.6, we set $k=2$ to ensure that relatively high trust is given to ray-paths that are associated with low scintillation risks.

So, given a hypothetical ground receiver and $S$ available satellites, different weights are assigned to the ray-paths (between the receiver and the satellites) based on the points the ray-paths pierce the ionospheric map. Then, WPDOP (Eq. 15) is estimated using the weighting matrix

where function $H_{d}$ is defined as

$$
\begin{aligned}
& H_{d}\left(x^{s}[j], \ldots, x^{s}\left[j+d_{k}+1\right] \mid v, \tau, x_{\mathrm{th}}\right) \\
& =\left\{\begin{array}{l}
1 \quad \text { if }\left(x^{s}[j], \ldots, x^{s}\left[j+d_{k}\right]\right) \geq x_{\mathrm{th}}, x^{s}\left[j+d_{k}+1\right]<x_{\text {th }} \text { and } x^{s}[j-1]<x_{\mathrm{th}} \\
\quad \text { when }\left\{\mathrm{v}^{s}[j-1], \ldots, \mathrm{v}^{s}\left[j+d_{k}+1\right]\right\} \in v \\
\left\{t_{j}, \ldots, t_{j+d^{k}+1}\right\} \in w 1 \text { if }\left(x^{s}[j], \ldots, x^{s}\left[j+d_{k}\right]\right) \geq x_{\mathrm{th}} \text { and } x^{s}[j-1]<x_{\mathrm{th}} \\
\quad \text { when }\left\{\mathrm{v}^{s}[j-1], \ldots, \mathrm{v}^{s}\left[j+d_{k}\right]\right\} \in v \text { and } \mathrm{v}^{s}\left[j+d_{k}+1\right] \notin v 1 \text { if }\left(x^{s}[j], \ldots, x^{s}\left[j+d_{k}\right]\right) \geq x_{\mathrm{th}} \\
\text { and } x^{s}\left[j+d_{k}+1\right]<x_{\mathrm{th}} \\
\text { when }\left\{\mathrm{v}^{s}[j], \ldots, \mathrm{v}^{s}\left[j+d_{k}+1\right]\right\} \in v \text { and } \mathrm{v}^{s}[j-1] \notin v 1 \text { if }\left(x^{s}[j], \ldots, x^{s}\left[j+d_{k}\right]\right) \geq x_{\mathrm{th}} \\
\text { when }\left\{\mathrm{v}^{s}[j], \ldots, \mathrm{v}^{s}\left[j+d_{k}\right]\right\} \in v \text { and } \mathrm{v}^{s}[j-1], \mathrm{v}^{s}\left[j+d_{k}+1\right] \notin v 0 \text { otherwise }
\end{array}\right.
\end{aligned}
$$

and gives either 1 or 0 based on the sequence $x^{s}[j-$ $1], \ldots, x^{s}\left[j+d_{k}+1\right]$.

Finally, by estimating pixel by pixel a risk (Eq. 17) at each $v$, we can obtain a map in matrix form denoted by

$R_{\text {ion }}^{\tau} \in \mathbb{R}^{V_{1} \times V_{2}}, \quad$ where $\left[V_{1} \times V_{2}\right]$

is the dimension of a uniform grid.

\subsection{WPDOP using scintillation risks}

To estimate WPDOP (Eq. 15) first we compute matrix $A \in$ $\mathbb{R}^{S \times 3}$ which includes the relative positions between a ground receiver and $S$ available satellites following the details of "Appendix A".

Regarding the weights, without further knowledge about the precise shape of function $h($ ) (the properties of which we anticipate may be gleaned from either statistical or physical information, or alternatively selected based on the requirements of a specific application), we approximate $w_{s}$ (Eq. 16) by

$w_{s}:=\left(1-r_{\text {ion }}^{(s)}\right)^{k}$,

where $r_{\text {ion }}^{(s)}$ is the ionospheric risk value at the intersection point between the line-of-sight of (link) $s$ and the iono-

$$
W=\left[\begin{array}{ccc}
\left(1-r_{\text {ion }}^{(1)}\right)^{k} & \cdots & 0 \\
\vdots & \ddots & \\
0 & & \left(1-r_{\text {ion }}^{(S)}\right)^{k}
\end{array}\right]
$$

Figure 3 b explains schematically how to assign a weight to each ray-path using the ionospheric risk map.

\section{Data, map construction, results and discussion}

In this section, we present ionospheric risk maps and corresponding WPDOP ground maps over the area of South America at geographic latitude between $\sim-40^{\circ} \mathrm{N}$ and $\sim 10^{\circ} \mathrm{N}$ and longitude between $\sim-90^{\circ} \mathrm{E}$ and $\sim-35^{\circ} \mathrm{E}$. This region experiences significant scintillation activity that causes deep signal fades inducing a GNSS receiver to lose lock of one or more satellite signals and includes the equatorial anomaly (APPLETON 1946).

\section{1 $\mathrm{S}_{4}$ data}

Scintillation activity maximizes mostly after sunset until a few hours after midnight local time during the equinoc- 
Fig. 2 a The ionospheric thin shell at $350 \mathrm{~km}$ was subdivided into a grid of spatial resolution $2^{\circ} \times 2^{\circ}$. The traces of all the projected $S_{4}$ samples (IPP data) on the ionospheric grid at 350 $\mathrm{km}$ over a period of $4 \mathrm{~h}(02$ November 2014) are depicted with black dots. The locations of scintillation monitors are denoted by blue triangles. $\mathbf{b}$ This figure depicts in higher resolution the square area marked with light blue colour in a. The traces of the projected $S_{4}$ samples that correspond to the same (satellite-scintillation monitor) link are marked with the same colour

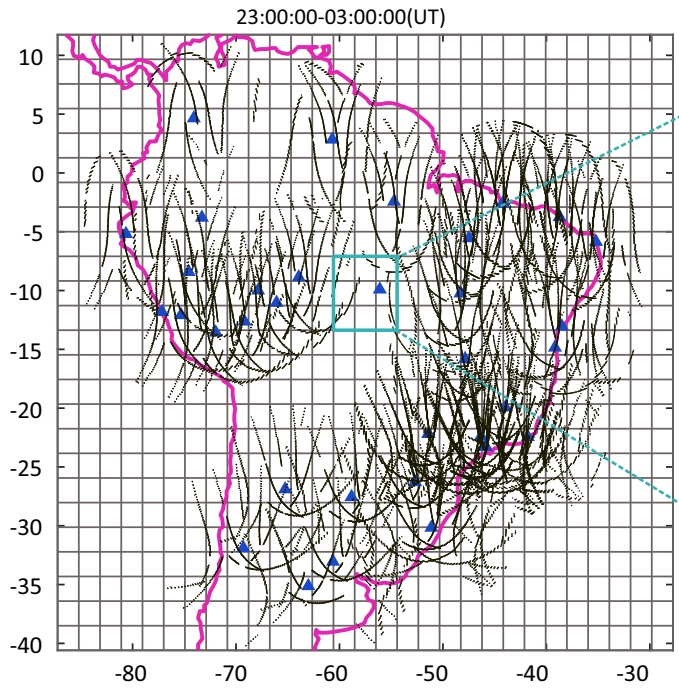

(a)

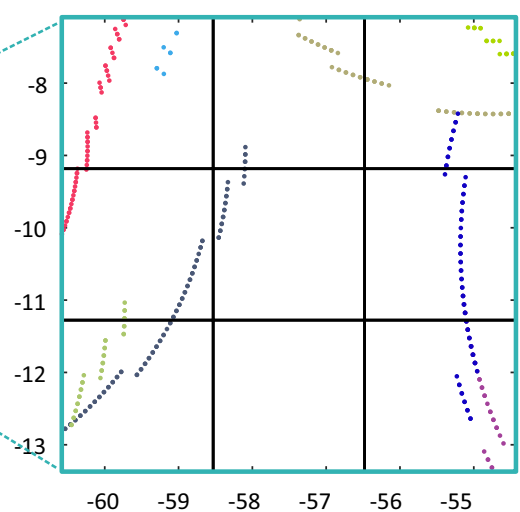

(b) tial months in the equatorial ionosphere (de Rezende et al. 2006). For this reason, in this study we used available $S_{4}$ data between 23.00 and 03.00 Universal Time (UT). The $S_{4}$ data (that were used to produce a risk map) were measured from a network of 38 ISMR scintillation monitors using all the available GPS, GLONASS and Galileo satellites over the 4-h period. In particular, the $S_{4}$ measurements ( with sampling period $\Delta=1 \mathrm{~min}$ ) and their corresponding IPPs at 350 $\mathrm{km}$ were provided from the CIGALA/CALIBRA networkUNESP web server (CIGALA/CALIBRA, b.d. 2009). The IPPS values for the interval between 23.00 and 03.00 (UT) are depicted with black dots in Fig. 2a. The period under investigation, which was during the night-time of 02 and 03 November 2014, was characterized by mild to strong scintillation according to the information provided by the web software (ISMR Query Tool) (http://is-cigala-calibra. fct.unesp.br) (Vani et al. 2017).

\subsection{Construction of ionospheric maps}

For the ionospheric risk maps, a uniform standard grid of spatial resolution $2^{\circ} \times 2^{\circ}$ was used for the estimation of the risk in each ionospheric pixel (as suggested also by (Takahashi et al. 2016)). A scintillation risk (joint probability Eq. 17) was estimated in each pixel using accumulated $S_{4}$ data over the interval 23.00-03.00 (UT) (for example, Fig. $2 b$ shows the available data over the 4-h interval for a set of nine pixels). Ionospheric pixels that did not have any IPP data were left blank in the following ionospheric maps.

For clarity in the subsequent text, the night-time interval (23.00-03.00 UT) crossing from 02 to 03 November 2014 will be simply referenced as that of 02 November 2014, and similarly, the night-time from 03 to 04
November 2014 will be referenced as that of 03 November 2014.

\subsection{Construction of ground maps}

The spatial resolution of the ground maps was selected as $1^{\circ} \times 1^{\circ}$ (in latitude and longitude) by placing hypothetical receivers (denoted by green triangles) in the centre of each pixel as shown in Fig. 3c. To calculate the WPDOP and PDOP for a set of hypothetical ground receivers, the positions of the satellites in view have to be estimated. There are different ways that this can be done using for example orbital information. In our study, the locations of the available satellites were computed geometrically at time $t$ using downloaded data from CIGALA/CALIBRA (b.d. 2009). Particularly, the downloaded data provided, in addition to $S_{4}$ measurements, the IPP geographic coordinates at $350 \mathrm{~km}$ (between the scintillation monitor and the satellite), the time of data acquisition and the satellite-monitor information (i.e. the PRN code of the satellite and name/location of the scintillation monitor) for each acquired $S_{4}$ measurement. Thus, the IPPs and locations of scintillation monitors that are associated with the same satellite at time $t$ were used to form 3D lines. The extensions of these lines were intersected at the location of the satellite (as depicted in Fig. 3a).

Furthermore, given the positions of the satellites at time $t$ over South America, the satellites in view for a hypothetical ground receiver were determined based on an elevation angle cut-off of $20^{\circ}$. Then, matrix $A$ in Eq. 15 was calculated using the locations of the satellites in view and the hypothetical receiver. For each (satellite-hypothetical receiver) line-ofsight (LoS), each weight (Eq. 25) in the diagonal matrix $W$ was computed using the risk value at the pixel where the line pierced the ionospheric map at $350 \mathrm{~km}$. Figure $3 \mathrm{~b}$ illustrates 


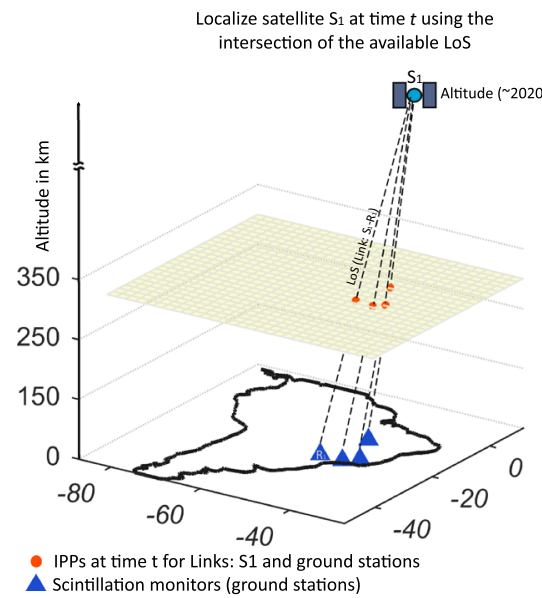

(a)

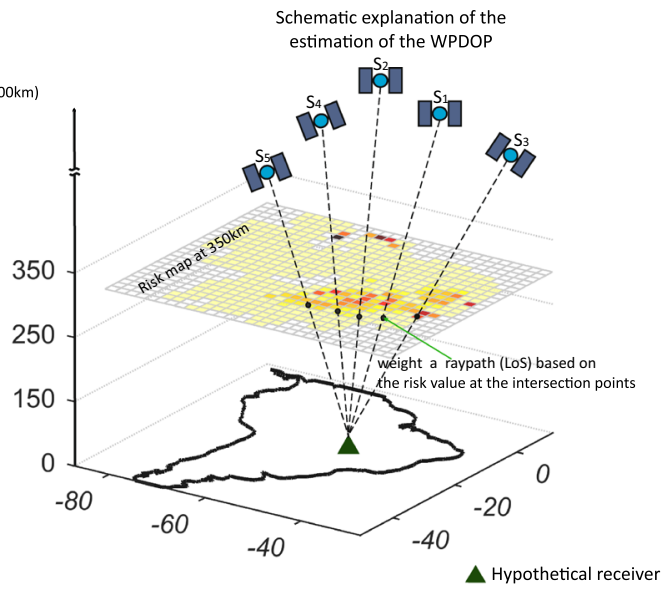

(b)

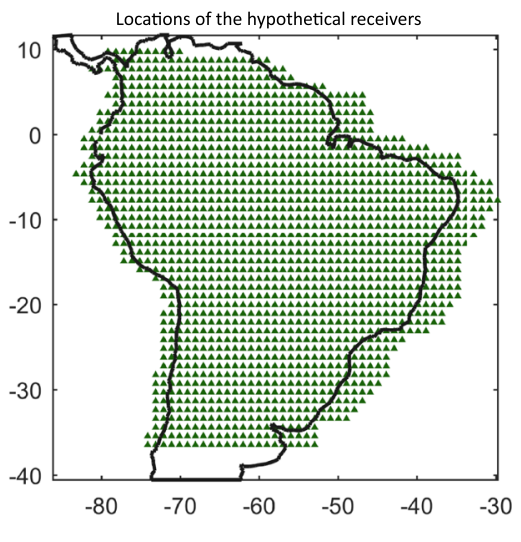

(c)
Fig. 3 a We localize a single satellite by estimating the intersection of line-of-sights (LoSs) that are formed by the ionospheric pierce points (IPPs) of $S_{4}$ samples and the locations of the scintillation monitors (denoted by blue triangles) at which the $S_{4}$ samples were recorded. b Schematic explanation of the estimation of the WPDOP for a hypothetical ground receiver. In this example, we have five available satellites which intersect the risk map at $350 \mathrm{~km}$ within five pixels marked with

an example of the estimation of the WPDOP for a hypothetical ground receiver when the ionospheric risk map is taken into account and five satellites are in view.

\section{4 lonospheric risk maps over South America}

Figures 4 and 5 show the ionospheric risk maps based on the joint probability of scintillation intensity and duration, using different thresholds for the $S_{4}$ value and duration of the scintillation event. More precisely, in Figs. 4 and 5 the rows correspond to $S_{4}$ thresholds $0.3,0.5$ and 0.7 , respectively, and the columns correspond to duration thresholds of 1, 5, $10 \mathrm{~min}$, respectively. The green line designates the geomagnetic equator, and the white pixels correspond to ionospheric regions where the risk was not estimated due to lack of data (the ionospheric regions where IPP data were available appear in the left image of Fig. 2 for 02 November 2014). As expected, based on the results of Fig. 4 we can observe that as the thresholds values are getting higher, the risk decreases.

Moreover, we can see that there are two bands of scintillation, i.e. north and south of the geomagnetic equator. This corresponds to the well-known equatorial anomaly (Kintner et al. 2007). The risk of scintillation in the north band seems to disappear for increasing thresholds relatively drastically (see second and third rows in Fig. 4) which indicates that scintillation of shorter duration and lower intensity prevails in this ionospheric region. We note that this could also be related to the number of the ground scintillation monitors small black dots. The weighting matrix in this example has the form $W=\operatorname{diag}\left(\left(1-r_{\text {ion }}^{(i)}\right)^{2}\right)$ where $r_{\text {ion }}^{(s)}$ are the risk values at the intersection points between the ray-paths $s$ and the risk map at $350 \mathrm{~km}$ (for $s=1, \ldots, 5)$. Then, matrix $W$ is inserted in the (Eq.15). c Green triangles show the locations of hypothetical receivers on the ground (used to estimate the dilution of precision ground maps)

which are less in the north band compared to the south band (so the south band may give us more reliable estimates).

In Fig. 5, scintillation structures remain similar as in Fig. 4; however, we can notice that the scintillation in Fig. 4 covers a broader area of the south band compared to Fig. 5 where highscintillation activity is observed predominantly over Brazil. Overall, we can say that scintillation of low intensity $(0.3 \leq$ $S_{4}<0.5$ ) affects a broad area of South America with risks approaching 0.8 , whereas scintillation risk drops drastically for $S_{4} \geq 0.7$ during both days.

\subsection{Ground maps over South America}

We illustrate the effect of scintillation on the ground by estimating the proposed weighted dilution of precision in a set of hypothetical ground receivers (small green triangles in Fig. 3c) using the risk maps estimated in the previous section and different sets of satellite constellations. Two representative examples of instant ground maps are shown in Figs. 6 and 7 for 02 November 2014 and 03 November 2014, respectively. For each day, the WPDOP and PDOP ground maps were calculated by utilizing the geometry of satellite constellations at precisely 01.00 .09 (UT). The weights for the WPDOP maps were based on the ionospheric risks maps estimated in Sect. 4.4 using $S_{4}$ data collected over 23.00 03.00 (UT). In particular, a weight (Eq. 25) for a link was computed using the risk value at the point where a receiversatellite ray-path pierces the ionospheric risk map (at 350 $\mathrm{km}$ ). The risk maps (a) and (e) were calculated considering 


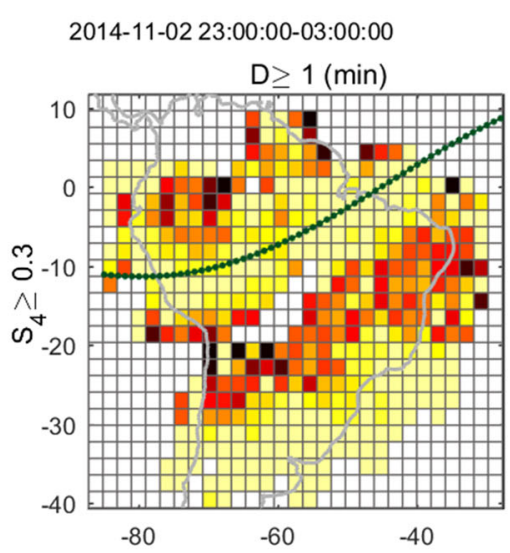

Ionospheric Scintillation Risk Maps: $\pi\left(D>d_{t h}, S_{4}>s_{t h}\right)$
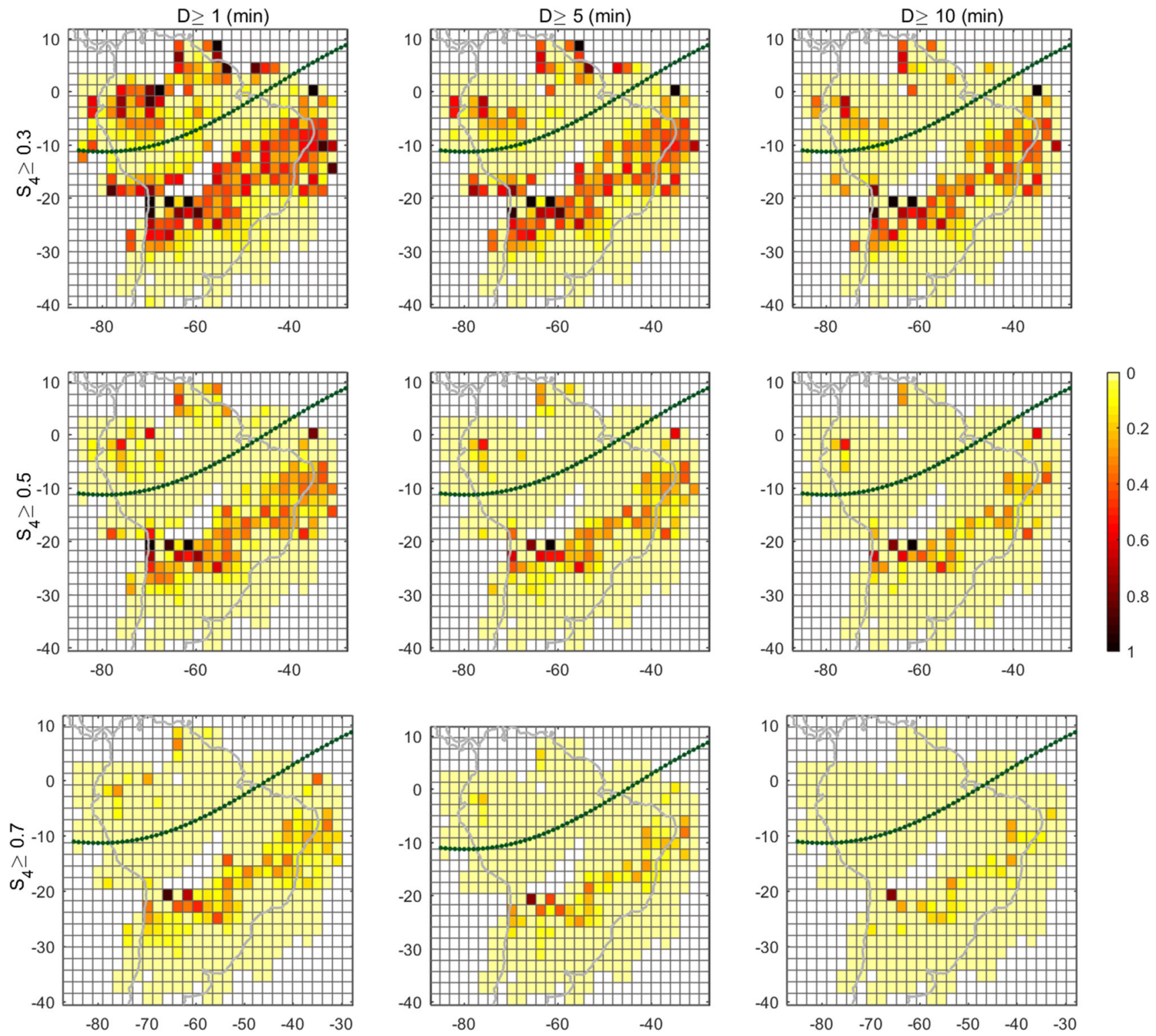

Fig. 4 Ionospheric risk maps for different $S_{4}$ thresholds and durations for 02 November 2014 (rows correspond to $S_{4}$ thresholds $0.3,0.5$ and

The risk maps (based on the joint probability Eq. 17) were estimated using accumulated $S_{4}$ over the interval 23.00-03.00 (UT). The dash green line depicts the geomagnetic equator

duration threshold of $5 \mathrm{~min}$ and $S_{4}$ intensity threshold either 0.3 or 0.5 (as shown in Figs. 6 and 7). Two sets of ground map estimations were computed to show the effects of scintillation with respect to the number of available constellations: (i) GPS (7 satellites) and (ii) GPS, GLONASS and Galileo (17 satellites). The elevation angle cut-off was set to $20^{\circ}$, i.e. raypaths which were lower than this threshold were discarded from the PDOP and WPDOP calculations.

In Fig. 6 (and similarly in Fig. 7), along column 1 we can observe the ionospheric risk maps; along column 2 , the maps of WPDOP estimated based on the risk maps (of column 1); along column 3 the map of PDOP (we note that maps (c) and (g) are identical and have been repeated to ease the comparisons); and along column 4 the contribution of scintillation to the total dilution of precision (in a percentage form). In Fig. 6i, PDOP values in the ground map (c) and (g) are low and increase slightly towards the west coast; in particular, in the area marked with the red ellipse the PDOP values are higher than in the corresponding area in Fig. 6ii (map (c) and (g)) due to less satellite coverage. In 


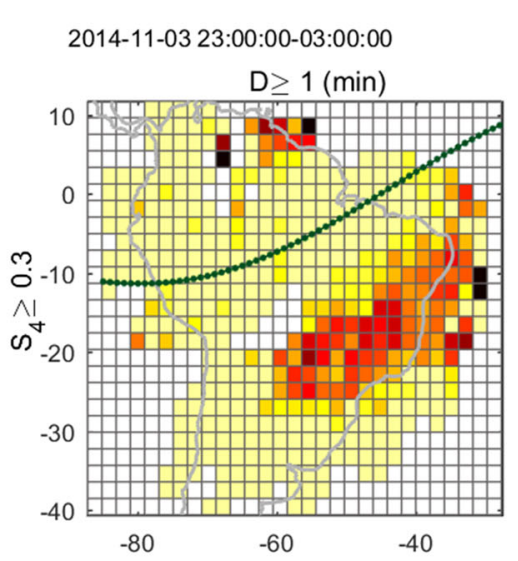

Ionospheric Scintillation Risk Maps: $\pi\left(D>d_{t h}, S_{4}>s_{t h}\right)$
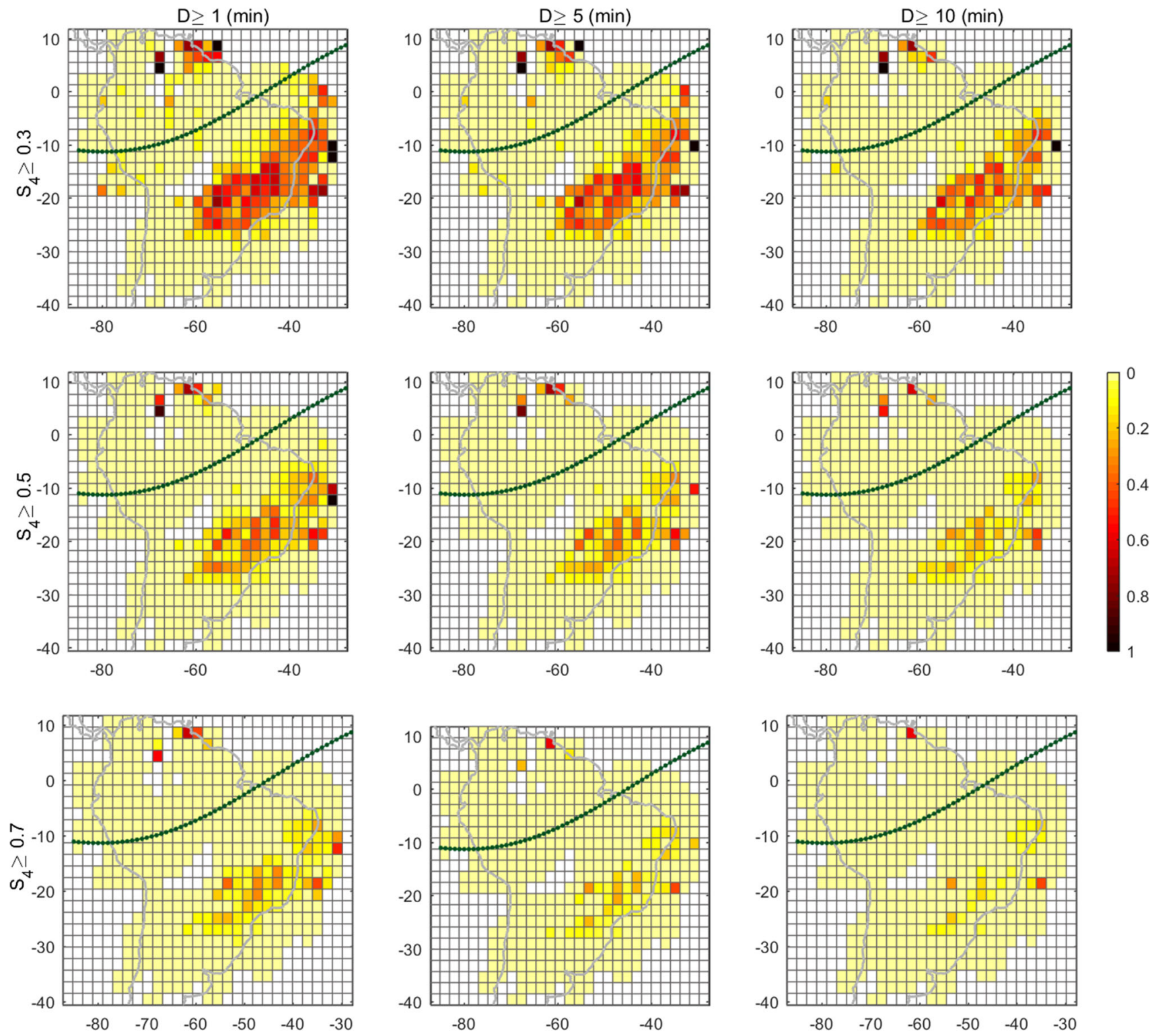

Fig. 5 Ionospheric risk maps for different $S_{4}$ thresholds and durations for 03 November 2014 (rows correspond to $S_{4}$ threshold 0.3, 0.5 and The risk maps (based on the joint probability Eq. 17) were estimated using accumulated $S_{4}$ over the interval 23.00-03.00 (UT)

general, WPDOP values (maps (b) and (f) along column 2) in Fig. 6i are increased mainly in the region under the scintillation structures. However, for longitude between $\sim-50^{\circ} \mathrm{E}$ and $\sim-40^{\circ} \mathrm{E}$ and latitude between $\sim-20^{\circ} \mathrm{N}$ and $\sim-15^{\circ} \mathrm{N}$, (i.e. the area marked with a pink ellipse in maps (b) and (f)) the WPDOP values are relatively low even though the risk due to scintillation over that region is significant. This is most likely because the ray-paths of the satellites in view (that were used to estimate the WPDOP at 01.00.09 (UT)) do not intersect the scintillation active region at this partic- ular moment. Moreover, in the north-west of the continent (area marked with blue circles in maps (b) and (f) of Fig. 6i), we can observe high WPDOP values. The higher values of WPDOP in that area (compared to the PDOP values in map (c) of Fig. $6 \mathrm{i}$ in the same area) indicate that $w_{s} \ll 1$. Low weights mean that the ray-paths between the hypothetical receivers located in the north-west and the satellites in view at 01.00.09 (UT) pierce the ionospheric map in pixels with high risk values based on Eq. 25 . 

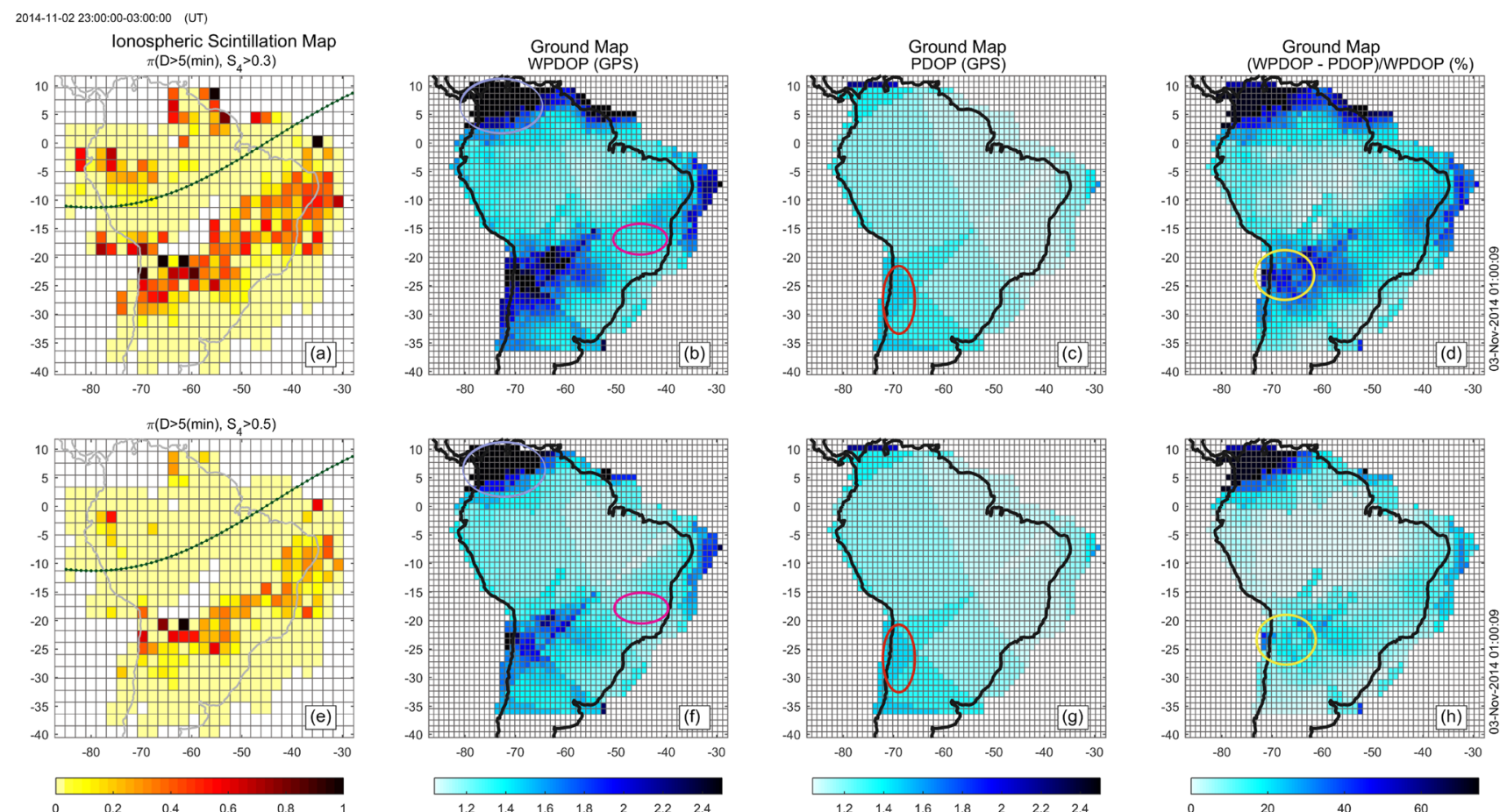

(i) Ionospheric risk map estimated using $S_{4}$ data over 23.00-03.00 (UT) and ground maps using only GPS constellation at 01.00 .09 (UT)

2014-11-02 23:00:00-03:00:00 (UT)

lonospheric Scintillation Map $\pi\left(\mathrm{D}>5(\mathrm{~min}), \mathrm{S}_{4}>0.3\right)$

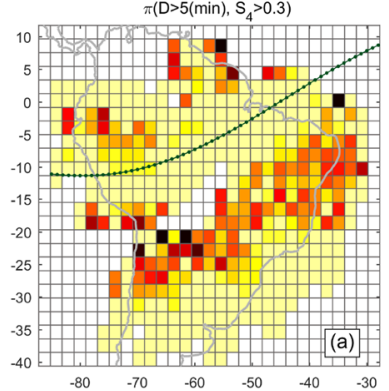

$\pi\left(\mathrm{D}>5(\mathrm{~min}), \mathrm{S}_{4}>0.5\right)$

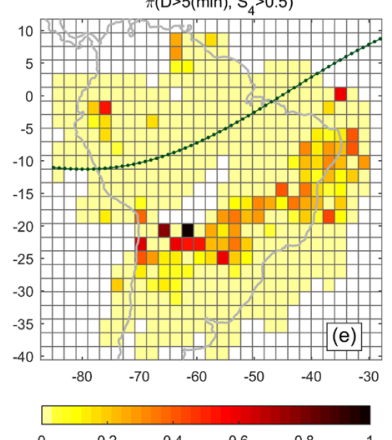

WPDOP Ground Map

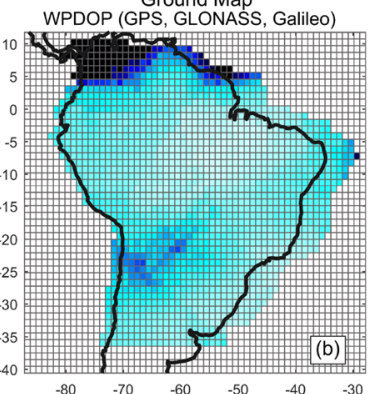

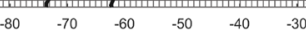

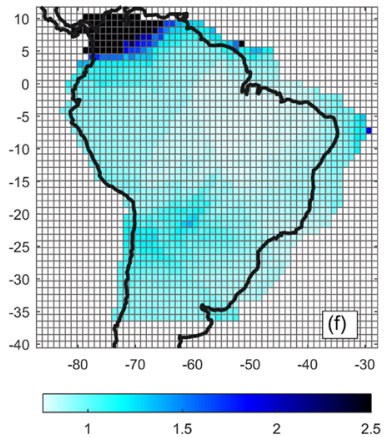

PDOP Ground Map

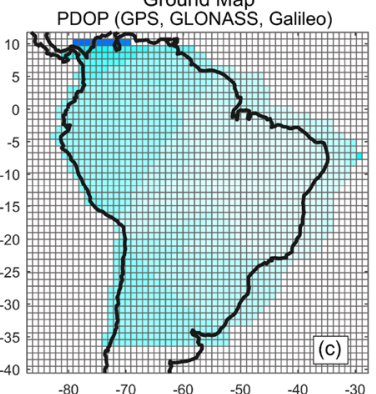

$\begin{array}{llllll}-80 & -70 & -60 & -50 & -40 & -30\end{array}$

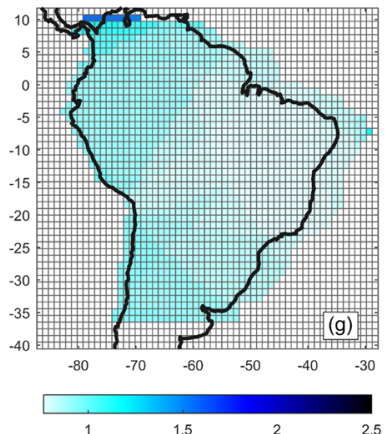

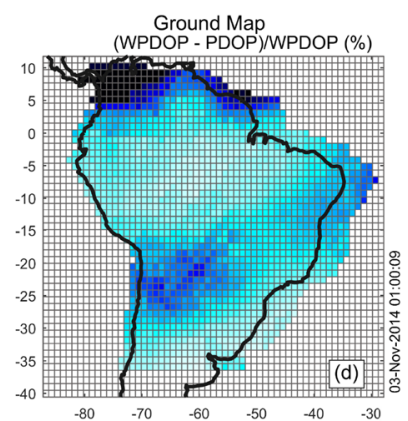

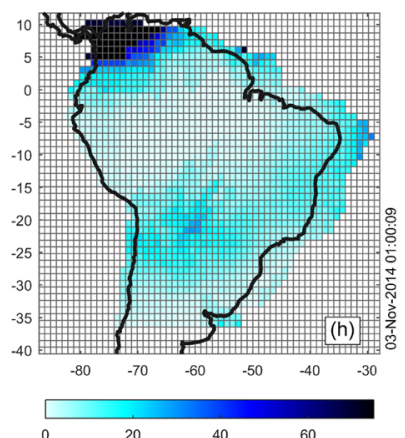

(ii) Ionospheric risk map using $S_{4}$ data over 23.00-03.00 (UT) and ground maps using GPS, GLONASS and Galileo constellation at 01.00.09 (UT)

Fig. 6 Risk maps and ground maps for different sets of satellite constellations for 02 November 2014. Ionospheric static risk maps were estimated using data over the interval 23.00-03.00 (UT). The geometry of the constellations for the ground maps was that of time instant 01.00.09 (UT). Within i or ii, each row corresponds to different thresholds in $S_{4}$. From left to right, column 1 shows ionospheric risk maps for thresholds in $S_{4}$ of 0.3 and 0.5 , and scintillation duration of $5 \mathrm{~min}$, col- umn 2 illustrates WPDOP ground maps which explicitly incorporate the scintillation risks as weights from ionospheric map in column 1, column 3 gives the PDOP ground maps (with no scintillation being included), and column 4 gives us the difference between WPDOP and PDOP, expressed as a percentage of WPDOP. The regions that are described in the text are marked with coloured ellipses 
WPDOP ground map (f) in Fig. 6i (column 2 and second row) reveals that high $S_{4}$ threshold (i.e. $S_{4} \geq 0.5$ ) reduces the effect of scintillation on the WPDOP values. This is because the strong scintillation activity is more focal based on the corresponding risk map (e) in Fig. 6i. However, by comparing the percentage maps (d) and (h) along the column 4 of Fig. 6i, we can observe that the loss in dilution of precision is localized similarly both when $S_{4} \geq 0.3$ and $S_{4} \geq 0.5$. Both (WPDOP-PDOP)/WPDOP maps of column 4 (in Fig. 6i) give a percentage of at least $30 \%$ contribution from scintillation which can be observed for longitude between $\sim-60^{\circ} \mathrm{E}$ and $\sim-75^{\circ} \mathrm{E}$ and latitude $\sim-20^{\circ} \mathrm{N}$ and $\sim-25^{\circ} \mathrm{N}$ (area marked with yellow ellipses in map (d) and (h)). This suggests that scintillation has a significant influence in the position accuracy and has to be taken into account. Furthermore, we can notice that further away from the ionospheric scintillation structures, the scintillation contribution to WPDOP drops to zero.

With the introduction of extra constellations, the effect of scintillation is reduced broadly with a small exception in the north part of the continent as we can observe in WPDOP maps (b) and (f) along column 2 of Fig. 6ii (area marked with an ellipse in red colour). This happens due to scintillation and not due to poor geometry since the PDOP values of the PDOP map (c) and (g) in column 3 of Fig. 6ii appear to be small in that region. In general, we can see along columns 4 of Fig. 6i, ii that the percentage contributions from scintillation, maps (d) and (h), are broadly similar which implies that while increasing the number of constellations (as currently shown) decreases the WPDOP values, the effect of scintillation is not totally eliminated.

Furthermore, test case of Fig. 7 demonstrates that the effect of scintillation activity can yield different ground structures. This is happening because the geometry of the available constellations and the risk maps are different between Figs. 6 and 7. The maps (d) and (h) along column 4 in Fig. 7 reveal "shadowing effects" on the Earth due to scintillation around the ionospheric active region, with the contribution decreasing with increasing distance from the scintillation structures. Similarly, as in the example of Fig. 7 we can conclude that the effect of scintillation on the ground is reduced when more satellites are in use and when the high-risk regions in the ionospheric map are more localized.

\subsection{Discussion}

\subsubsection{Current implementation and intrinsic limitations}

In the current application of our methodology, we were restricted by the number of available ground scintillation monitors (unfortunately, at the moment the ground monitors are very few and unevenly spread over the continent) and the fixed number of satellites. So, in effect these lim- itations forced us to produce ionospheric scintillation risk maps using data collected over a relatively long time window. The selection of the 4-h time window (period of the highest scintillation activity) was used to capture the average scintillation activity. Therefore, the estimated static risk maps described the average scintillation activity during the specific time window.

A WPDOP value was estimated for a specific location using the number of available satellites at that moment and the risk maps, as shown in Fig. 3b. The weights were assigned to different ray-paths based on the ionospheric scintillation risk maps and were directly related to statistical information about scintillation since the risks that were used to estimate the weights (Eq. 26) had been computed from scintillation data (i.e. scintillation intensity and duration which has been extracted from $S_{4}$ measurements). Therefore, the proposed WPDOP included the impact of the average scintillation activity.

In the future, if more scintillation monitors are built on the ground, then our methodology can produce ionospheric scintillation maps with higher temporal resolution (say, every 30 or $15 \mathrm{~min}$ ). This will allow to update the weights in the WPDOP constantly and to observe the impact of the dynamic scintillation activity. Currently, we can say that a moving window and/or a shorter time window for the data collection (to construct ionospheric risk maps) could be implemented only in areas that are covered by a dense network of scintillation monitors. By using shorter data collection intervals and considering, for example, lower scintillation thresholds, we could produce scintillation risk maps useful in aircraft navigation. These risk maps can update the weights in the PDOP and provide information to the pilot whether to trust the navigation system.

\subsubsection{Comparing WPDOP with PDOP}

The dilution of precision is used as a quality measure in positioning systems since it expresses the expected uncertainty in the positioning estimates. Roughly speaking, a low dilution of precision implies high confidence level in the positioning results, and a high dilution of precision indicates low confidence level.

In general, we can notice that the standard PDOP considers implicitly the scintillation effects in the sense that strong scintillation can lead to loss of one or more satellites resulting in high measured PDOP values (due to poor satellite geometry of the remaining satellites). However, by using the standard PDOP it is not possible to quantify the impact that low-to-high-scintillation activity has on the accuracy of a positioning system when losses of lock do not occur. In such cases, the degradation of the GNSS positioning may be significant even though the satellite constellation geometry would appear promising. The concept of WPDOP allows to 
2014-11-03 23:00:00-03:00:00 (UT)

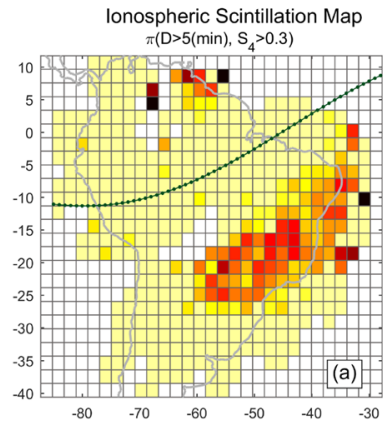

$\pi\left(\mathrm{D}>5(\mathrm{~min}), \mathrm{S}_{4}>0.5\right)$
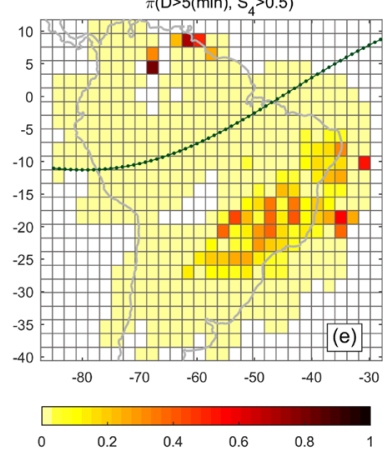

2014-11-03 23:00:00-03:00:00 (UT)

Ionospheric Scintillation Map $\pi\left(\mathrm{D}>5(\mathrm{~min}), \mathrm{S}_{4}>0.3\right)$

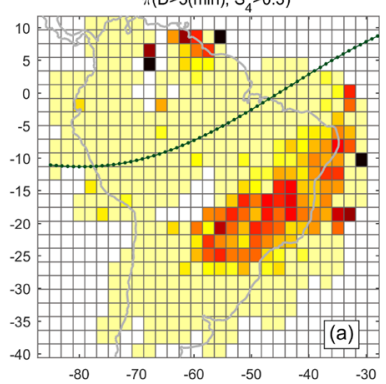

$\pi\left(\mathrm{D}>5(\min ), \mathrm{S}_{4}>0.5\right)$

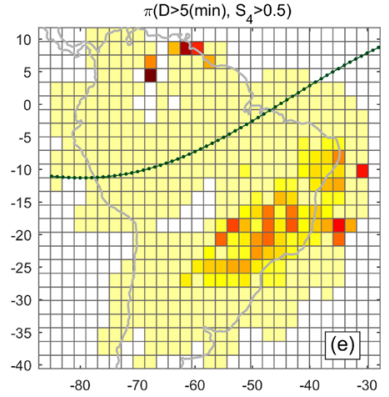

\begin{tabular}{llll}
\hline 0.2 & 0.4 & 0.6 & 0.8
\end{tabular}
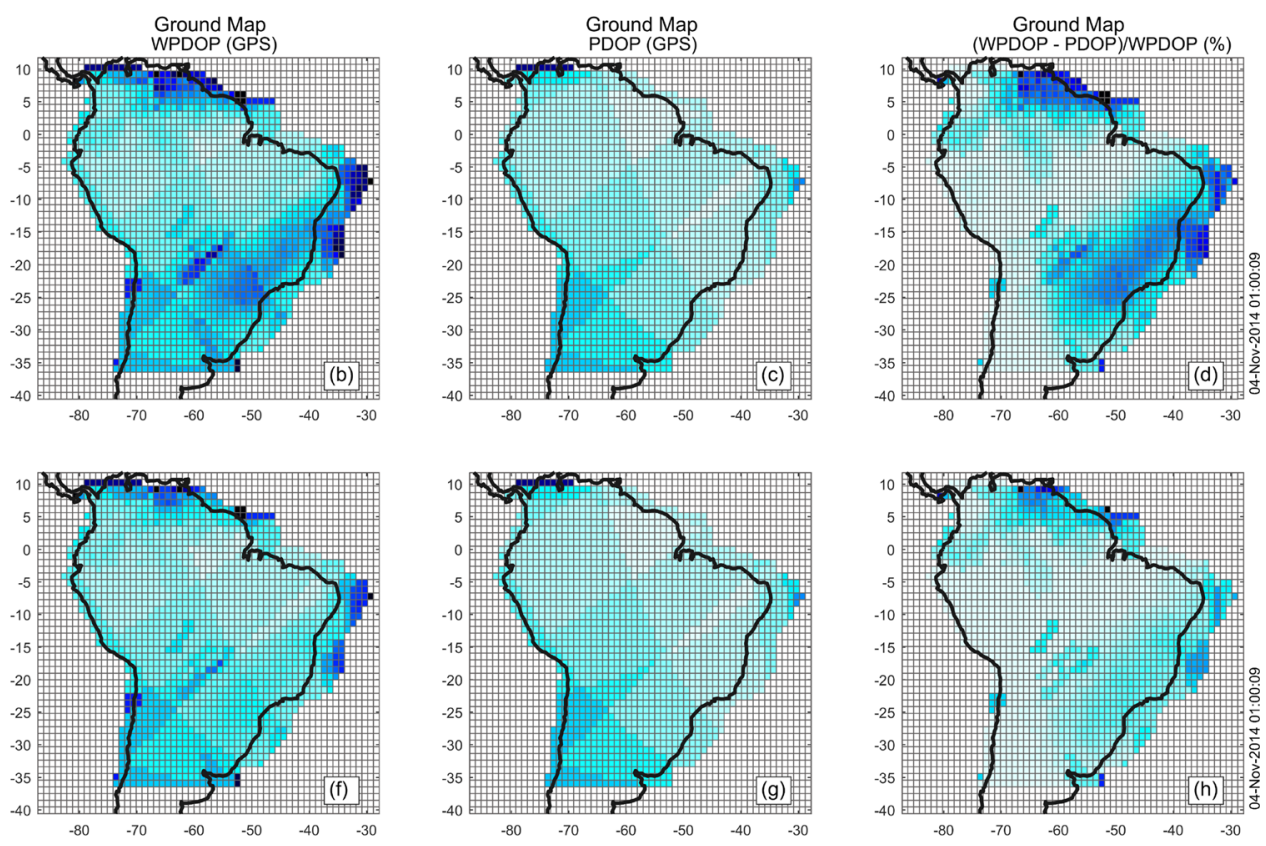

$\begin{array}{lllllll}1.2 & 1.4 & 1.6 & 1.8 & 2 & 22 & 2.4\end{array}$

$\begin{array}{lllllll}12 & 1.4 & 16 & 18 & 2 & 22 & 24\end{array}$

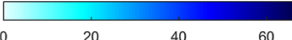

(i) Ionospheric risk map estimated using $S_{4}$ data over 23.00-03.00 (UT) and ground maps using only GPS constellation at precisely 01.00.09 (UT)
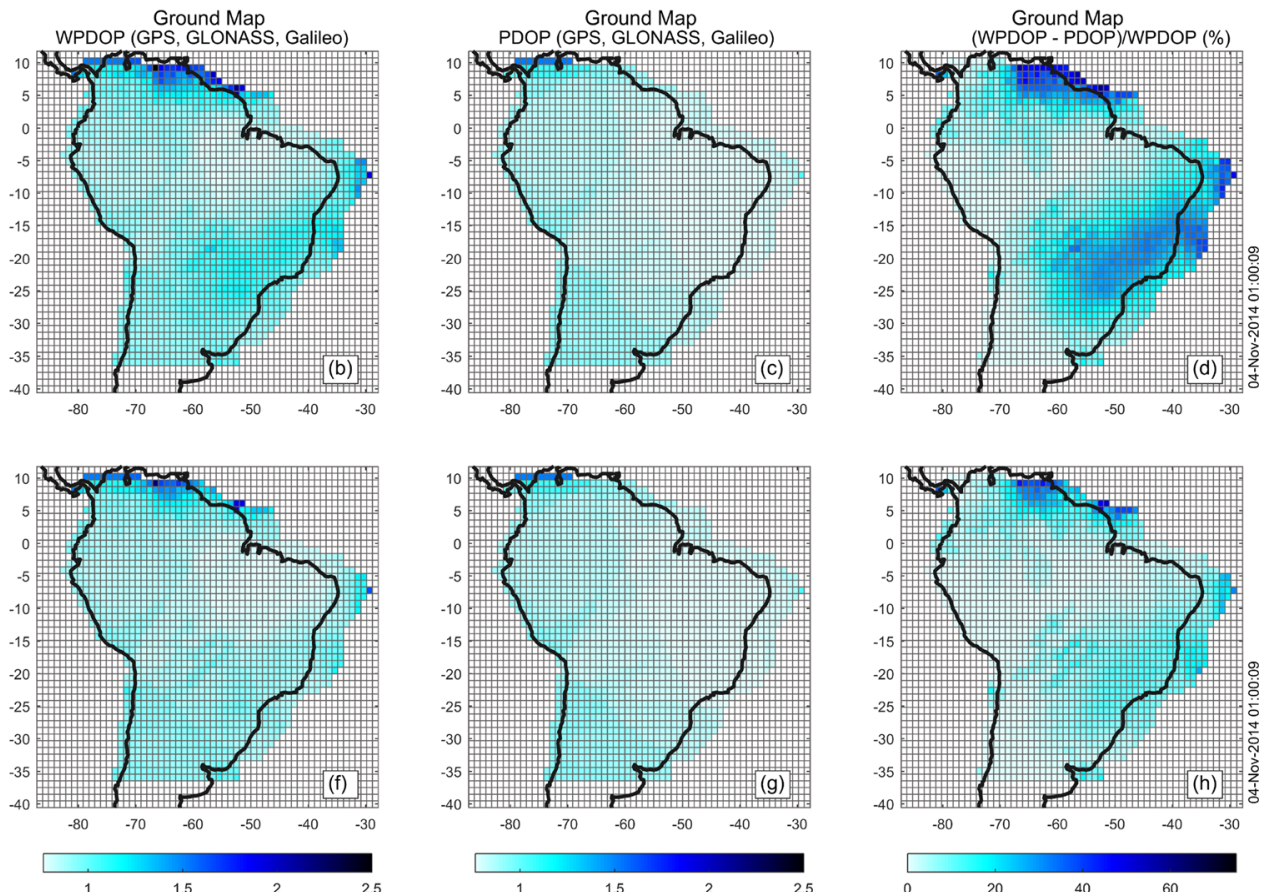

(ii) Ionospheric risk map using $S_{4}$ data over 23.00-03.00 (UT) and ground maps using GPS, GLONASS and Galileo constellation at 01.00.09 (UT)

Fig. 7 The results are presented as in Fig. 6. Ionospheric risk maps and ground maps for different sets of satellite constellations for 03 November 2014 
estimate the impact that scintillation-induced errors can have on PDOP by assigning the proposed scintillation weights in all the available satellite-receiver links.

Overall, we observed that the more satellites were used in the estimation of the dilution of precision, the lower were the values both for PDOP and WPDOP and hence lower position errors were expected. ${ }^{5}$ So, the combination of GPS, GLONASS and Galileo can improve precision. However, there were still some areas which were affected by scintillation (especially for weak scintillation of duration $5 \mathrm{~min}$ or more) even when all the available constellations were used. This suggests that by increasing the number of satellites in view, the positioning precision can be further improved in the case of strong localized scintillation. This outcome is in line with a recent work by Marques et al. (2018) and the review article by Langley (2012).

\subsubsection{Novelty and transferability}

In this work, instead of using basic statistical analysis, we exploited the concept of risk from decision theory to quantify the effects that the ionospheric scintillation can have on potential end-user activities. In particular, we derived risks considering that the quality of a satellite-receiver communication can be characterized only by scintillation measurements and then we constructed ionospheric risk maps using $S_{4}$ data provided by the UNESP server in South America. As we showed in Sect. 4.4, this methodology can be used for the construction of sequences of ionospheric risk maps (Figs. 4 and 5). Such maps could be further used as a priori information for ionospheric stochastic models, for enriching scintillation climatology databases or for improving understanding of scintillation phenomena over multiple years (Priyadarshi 2015; Jiao et al. 2018). Moreover, by explicitly incorporating scintillation risks into dilution of precision, one can overcome intrinsic limitations of empirical models and receiver specifications. Finally, the proposed methodology allows to produce high-resolution ground maps which could potentially be used to help mitigate the effects of ionospheric scintillation on GNSS positioning.

The proposed methodology for the construction of ionospheric risk and ground maps is reusable and transferable. Notably, if one wants to tailor risk to specific applications, then this methodology has the advantage that its parameters can be optimized and validated for specific applications. For example, the loss function (Eq. 4) allows us to select scintillation intensity and duration thresholds, $x_{\mathrm{th}}$ and $d_{\mathrm{th}}$, respectively, based on the specifications of the receiver and the needs of safety critical applications. By employing a $0-1$ loss function, we showed that risks can be easily interpreted

\footnotetext{
${ }^{5}$ For further details about the link between WPDOP/PDOP and position error, see "Appendix C".
}

in terms of probability of scintillation events. However, not only the thresholds, but also alternative loss functions to Eq. 4 can be adopted. Moreover, our risk formalism allows to consider extra (measurable) inputs in feature $Z$ (of Sect. 2.1) in addition to scintillation data $X$ and durations of events $D$, such as information about data gaps and other ionospheric quantities (e.g. total electron content (TEC)). Therefore, the risks (Eq. 5) (as joint probabilities of multiple parameters) can be estimated to illustrate an overall ionospheric activity. Regarding the weighting factors $w_{s}$ (Eq. 16), different shapes of the error variance function $h$ (.) (Eq. 11) can be adopted based on the outcomes of future research without any changes in the proposed methodology of Sect. 2.3.

Alternatively, the construction of multiple risk maps using different sets of inputs (measurable features), such as scintillation values measured in different frequencies which reflect how small-scale plasma density irregularities in F-region affect different propagating signals and gradients of the TEC which can capture large-scale irregularities, can offer versatile and complimentary information to the users. As a further step, these sets of risk maps can infer implicit statistical information about different (multi-frequency) errors which can be considered in the position error model (Eq. 6) and the estimation of the weights in the WPDOP. Hence, the concept of WPDOP can be extended to include other ionosphericrelated errors to inform how different propagation errors arising from ionospheric structures can impact the positioning on the ground.

In particular, our formalism enables the adaptation of the WPDOP given the presence of scintillation or any other ionospheric-related errors (e.g. in the equatorial F-region), For example, the error term in the observation model (Eq. 6) could be decomposed into more uncorrelated error terms, e.g. $\varepsilon=\varepsilon_{\mathrm{sc}}+\varepsilon_{\Delta \mathrm{TEC}}+\varepsilon_{\mathrm{rem}}$ and the corresponding ionospheric risks could be used to provide information about the different error variances (expressed as weights) for the WPDOP, e.g.

$w_{s}=\frac{\gamma}{\gamma_{\varepsilon^{(s)}}}=\frac{\gamma}{\gamma+h\left(r_{\mathrm{sc}}^{(s)}\right)+g\left(r_{\Delta \mathrm{TEC}}^{(s)}\right)}$ for $s=1 \ldots S$,

where $g\left(r_{\Delta \mathrm{TEC}}^{(s)}\right)$ is the error variance due to $\triangle \mathrm{TEC}$ ionospheric effects. Therefore, based on the available statistical knowledge, a WPDOP calculated by any hypothetical receiver could depend upon the combination of scintillation levels on multiple frequencies (utilized for positioning), the presence of losses of lock on some or all the frequencies associated with a given link, other ionospheric errors (e.g. TEC) and the geometry of the available links. Then, by comparing WPDOP against PDOP, we can infer information about the impact that different ionospheric irregularities can have on the positioning accuracy on the ground. 


\section{Conclusions}

This paper proposed a methodology which connects the ionospheric uncertainties in the sky originating from small-scale irregularities in the F-region (e.g. in the equatorial ionosphere) with their impact on the positioning on the ground. In particular, first we exploited decision theory to develop risks defined as expected losses that incur during a satellite communication activity which is assumed to be fully characterized by ionospheric measurable features, in our case scintillation intensity and duration. The proposed scintillation risks relied on a $0-1$ loss function, and thus, they were equal to the joint probability of scintillation intensity and duration above specified thresholds. The derived risk formulation was used to estimate pixelwise risk values in a uniform ionospheric grid at $350 \mathrm{~km}$ and the estimated risk maps reflected the ionospheric plasma density inhomogeneities causing scintillation during the observed time window.

We demonstrated our methodology by estimating risk maps in the sky using accumulated data obtained from GPS, GLONASS and Galileo scintillation monitors in South America during local night-time hours and using different scintillation intensity and duration thresholds. Overall, we observed that the two bands of scintillation, i.e. north and south of the geomagnetic equator, were visible in cases where the intensity threshold did not exceed value 0.5 and when the duration of the scintillation events was up to 10 min. Subsequently, to understand the effect of scintillation on the ground, we proposed to use a weighted position dilution of precision (WPDOP) which was estimated by assigning different weights to receiver measurements from different satellites using the ionospheric scintillation risks along the line-of-sights (LoSs) of the corresponding links. Thus, the dilution of precision on the ground depended upon the combination of the geometry of the available links and the scintillation-induced observable errors (quantified through the scintillation risks) on the same available links.

Finally, we constructed instantaneous WPDOP ground maps combining information from the ionospheric risk maps and the available link geometries. The WPDOP maps revealed those areas on the ground which were more affected by ionospheric scintillation. We noted that the scintillation effects on the ground receiver were more prominent when only GPS was available. However, the effects were not totally eliminated with the addition of extra constellations.

The present work focused on the effect of ionospheric scintillation to the dilution of precision. In the future, other sources of errors or modelling uncertainties can be taken into account in the dilution of precision estimations (i.e. super- position of risks associated, not only with scintillation but also with other ionospheric measurable features, can be considered). Also, we expect to further study how errors due to scintillation limit the accuracy in position estimation from both theoretical and applied perspectives and how statistical information about these errors can be incorporated into existing precise point positioning softwares.

Acknowledgements This work was supported by the Natural Environment Research Council (NERC), UK, (NE/R009082/1). AK was supported by the Academy of Finland Postdoctoral Researcher program (No. 316542). BV thanks Federal Institute of Education, Science and Technology of Sao Paulo (IFSP), Sao Paulo State University (UNESP) and CAPES (CAPES/PDSE n. 19-2016/Process No. 88881.134266/2016-01) for supporting his research. The authors would like to thank Prof. J. F. G. Monico, Department of Cartography, Sao Paulo State University (UNESP) and Prof. M. H. Shimabukuro, Department of Computer Science, Sao Paulo State University (UNESP) for providing the $S_{4}(\mathrm{~L} 1)$ data that were deployed in the context of the Projects CIGALA and CALIBRA (both funded by the European Commission (EC) in the framework of the FP7-GALILEO-2009-GSA and FP7GALILEO2011GSA1a, respectively), and FAPESP Project No. 06/04008-2. The ground stations and $S_{4}$ data, that were used to demonstrate the proposed methodology, are currently maintained by the National Institute of Science and Technology_GNSS Technology to Support Air Navigation (INCT GNSS-NavAer), funded by CNPq (National Council for Scientific and Technological Developmentprocess 465648/2014-2), FAPESP (Sao Paulo Research Foundationprocess 2017/01550-0) and CAPES (Coordination for the Improvement of Higher Education Personnel).

Author contributions For ionospheric maps, AK, NDS and BCV designed the methodology with the support from the other authors; For ground maps AK and DS formulated the theory with support from VR; $\mathrm{AK}$ and $\mathrm{BCV}$ implemented the ionospheric maps; $\mathrm{AK}$ implemented the ground maps; AK and NDS analysed the results; AK wrote the paper as main author in collaboration with NDS and VR and with editorial support from BCV, IA and BF; BF and IA were involved in scoping the research and ongoing discussion.

Data Availability Statement The $S_{4}$ data used to estimate the ionospheric maps in Sect. 4.4 were downloaded from http://is-cigala-calibra. fct.unesp.br; The MATLAB ${ }^{\circledR}$ codes for the estimation of the ionospheric and ground maps can be downloaded from the link https:// github.com/AlexandraKoulouri/Ionospheric-Scintillation-Maps-andPDOP.

Open Access This article is licensed under a Creative Commons Attribution 4.0 International License, which permits use, sharing, adaptation, distribution and reproduction in any medium or format, as long as you give appropriate credit to the original author(s) and the source, provide a link to the Creative Commons licence, and indicate if changes were made. The images or other third party material in this article are included in the article's Creative Commons licence, unless indicated otherwise in a credit line to the material. If material is not included in the article's Creative Commons licence and your intended use is not permitted by statutory regulation or exceeds the permitted use, you will need to obtain permission directly from the copyright holder. To view a copy of this licence, visit http://creativecomm ons.org/licenses/by/4.0/. 


\section{A Pseudorange measurements for position recovery}

The pseudorange observation model (at frequency $\left(f_{i}\right)$ in the $L_{i}$ band can be written as (Langley 1999; Marques et al. 2018),

$P^{(s)}(r)=\left\|r-r^{(s)}\right\|_{2}+v$,

where $P^{s}(r)$ is the measured pseudorange, $\left\|r-r^{(s)}\right\|_{2}=$ $\sqrt{\left(r_{x}-r_{x}^{(s)}\right)^{2}+\left(r_{y}-r_{y}^{(s)}\right)^{2}+\left(r_{z}-r_{z}^{(s)}\right)^{2}}$ is the geometric range between the receiver's antenna at location $r=$ $\left(r_{x}, r_{y}, r_{z}\right)$ and the satellite's antenna at location $r^{(s)}=$ $\left(r_{x}^{(s)}, r_{y}^{(s)}, r_{z}^{(s)}\right)$, respectively. Term $v$ represents the model errors and any unmodelled effects including the clock errors. We note that in the current analysis, we omit the clock uncertainty effect and thus to retrieve receiver's position, $r$, we need to have a set of at least three equations of the form (Eq. 27); in other words, at least 3 satellites have to be available.

Since the observation model (Eq. 27) is nonlinear, a firstorder Taylor approximation is used, i.e.

$P^{(s)}(r)=P^{(s)}\left(r_{0}\right)+\left(\nabla P^{(s)}\left(r_{0}\right)\right)^{\mathrm{T}} \Delta r+\varepsilon^{(s)}$,

where $P^{(s)}\left(r_{0}\right)$ is an approximate (computed) estimate, $\nabla P^{(\mathrm{s})}\left(r_{0}\right)=\left[\frac{\partial P^{(s)}}{\partial r_{x}}, \frac{\partial P^{(s)}}{\partial r_{y}}, \frac{\partial P^{(s)}}{\partial r_{z}}\right]^{\mathrm{T}}, \Delta r=\left[\Delta r_{x}, \Delta r_{y}, \Delta r_{z}\right]^{\mathrm{T}}$ is the position error, and $\varepsilon^{(s)}$ includes all the errors related to the numerical approximations and the model uncertainties. Furthermore, based on Eq. 27, $\nabla P^{(s)}\left(\mathrm{r}_{0}\right)=\left[\frac{r_{x_{0}}-r_{x}^{(s)}}{\left\|\mathrm{r}_{0}-\mathrm{r}^{(s)}\right\|_{2}}\right.$, $\left.\frac{r_{y_{0}}-r_{y}^{(s)}}{\left\|\mathrm{r}_{0}-\mathrm{r}^{(s)}\right\|_{2}}, \frac{r_{z_{0}}-r_{z}^{(s)}}{\left\|\mathrm{r}_{0}-\mathrm{r}^{(s)}\right\|_{2}}\right]^{\mathrm{T}}$.

Then, we end up with a set of linear equations

$b=A \Delta r+\varepsilon$,

where $b=\left[P^{(1)}(r)-P^{(1)}\left(r_{0}\right), \ldots, P^{(S)}(r)-P^{(S)}\left(r_{0}\right)\right]^{\mathrm{T}} \in$ $\mathbb{R}^{S}, A=\left[\nabla P^{(1)}\left(r_{0}\right) \ldots \nabla P^{(S)}\left(r_{0}\right)\right]^{\mathrm{T}} \in \mathbb{R}^{S \times 3}$ and $\varepsilon \in \mathbb{R}^{S}$. Here, we assume that even in the absence of a loss of lock, scintillation can induce higher-order errors in the observables that do not cancel out in dual-frequency combinations (Aquino et al. 2009; van den IJssel et al. 2016). Eq. 29 applies for both single-frequency positioning and dual-frequency positioning.

\section{B Defining the position dilution of precision}

As we will show in the following text, the position dilution of precision is a measure of the uncertainty of the estimates
$\widehat{\Delta r}$ and it is parameterized according to the statistical characterization of the errors $\varepsilon$ and the linear model used for the position estimates (Eq. 6). In particular, by denoting $\Gamma_{x x}$, $\Gamma_{y y}$ and $\Gamma_{z z}$ the diagonal elements of the general covariance $\Gamma=\left(A^{\mathrm{T}} \Gamma_{\varepsilon}^{-1} A\right)^{-1}$ (Eq. 14), the standard deviations of $\widehat{\Delta r}$ (Eq. 13) in X, Y and Z directions are $\sigma_{x}=\sqrt{\Gamma_{x x}}, \sigma_{y}=\sqrt{\Gamma_{y y}}$ and $\sigma_{z}=\sqrt{\Gamma_{z z}}$, respectively. The dilution of precision is defined as the normalized (with a common scaling factor $\kappa$ ) root mean square (RMS) of the standard deviations given by

$$
\begin{aligned}
\mathrm{RMS} & =\sqrt{\sigma_{x}^{2}+\sigma_{y}^{2}+\sigma_{z}^{2}}=\sqrt{\Gamma_{x x}+\Gamma_{y y}+\Gamma_{z z}} \\
& =\sqrt{\operatorname{tr}(\Gamma)}=\sqrt{\operatorname{tr}\left(\left(A^{\mathrm{T}} \Gamma_{\varepsilon}^{-1} A\right)^{-1}\right)} .
\end{aligned}
$$

So, the general form of the position dilution of precision is

$$
\begin{aligned}
\operatorname{PDOP}_{X Y Z} & =\frac{\mathrm{RMS}}{\sqrt{\kappa}} \\
& =\frac{\sqrt{\operatorname{tr}\left(\left(A^{\mathrm{T}} \Gamma_{\varepsilon}^{-1} A\right)^{-1}\right)}}{\sqrt{k}}=\sqrt{\operatorname{tr}\left(\left(A^{\mathrm{T}} W A\right)^{-1}\right)}
\end{aligned}
$$

where $W=\left(\frac{\Gamma_{\varepsilon}}{\kappa}\right)^{-1}$. Based on that ratio, we can see that the weights can be derived based on the prior knowledge about the error statistics. Furthermore, from the expression of the PDOP $_{X Y Z}$ we can understand that the dilution of precision is not the actual positioning error, but a measure of the uncertainty of the estimates $\widehat{\Delta r}$. Therefore, the key feature of the dilution of precision is that it is a single value that can be used as an indicator about the reliability of a positioning system. Moreover, we can notice that as matrix $A$ and $W$ do not depend on the measurements, but only on the geometry and the weighting scheme, dilution of precision can be computed from the satellite orbital information without needing real measurements. Currently, there are empirical tables that relate PDOP values with the expected accuracy of a positioning system.

In the following text, we will see that the scaling factor $\kappa$ is defined based on the model of the observation error. More precisely, for observation errors modelled as in Sect. 2.3.1, the error covariance is given by $\Gamma_{\varepsilon}=\gamma I^{S \times S}$ and

$\mathrm{RMS}=\sqrt{\gamma \operatorname{tr}\left(\left(A^{\mathrm{T}} A\right)^{-1}\right)}$.

Therefore, the common scaling factor is $\kappa=\gamma$ and $W=$ $I^{S \times S}$. Then, we have the well-known standard position dilution of precision given by Eq. 9 .

For an observation error modelled as in Eq. 10 of Sect. 2.3.2, the error covariance has two terms, i.e.

$\Gamma_{\varepsilon}=\gamma I^{S \times S}+\Gamma_{\varepsilon_{\mathrm{sc}}}$. 
Using the matrix inversion lemma, we have that $\Gamma_{\varepsilon}^{-1}=$ $\gamma^{-1}\left(I^{S \times S}-\left(\gamma \Gamma_{\varepsilon_{\mathrm{sc}}}^{-1}+I^{S \times S}\right)^{-1}\right)$. Inserting the previous expression in the RMS, we have that

$\operatorname{RMS}=\sqrt{\gamma \operatorname{tr}\left(\left(A^{\mathrm{T}} U A\right)^{-1}\right)}$,

where $U=I^{S \times S}-\left(\gamma \Gamma_{\varepsilon_{\mathrm{sc}}}^{-1}+I^{S \times S}\right)^{-1}$ and $\Gamma_{\varepsilon}^{-1}=\gamma^{-1} U$. Therefore, the scaling factor $\kappa=\gamma$ and now $W=U$. Given the scintillation error covariance $\Gamma_{\varepsilon_{\mathrm{sc}}}=\operatorname{diag}\left(h\left(r_{\text {ion }}^{(s)}\right)\right)$ we can easily show that $W=\operatorname{diag}\left(\frac{\gamma}{\gamma+h\left(r_{\text {ion }}^{(s)}\right)}\right)$ as in Eq. 16. Therefore, based on the previous error analysis, PDOP is given by

$\operatorname{PDOP}_{X Y Z}=\frac{\sqrt{\sigma_{x}^{2}+\sigma_{y}^{2}+\sigma_{z}^{2}}}{\sqrt{\gamma}}=\frac{\sqrt{\operatorname{tr}\left(\left(A^{\mathrm{T}} \Gamma_{\varepsilon}^{-1} A\right)^{-1}\right)}}{\sqrt{\gamma}}$.

\section{Average position error and position dilution of precision}

Let us first clarify that $\Delta r=r_{\mathrm{tr}}-r_{0}$ is the position difference between the true XYZ position vector, $r_{\mathrm{tr}}=\left(r_{x_{\mathrm{tr}}}, r_{y_{\mathrm{tr}}}, r_{z_{\mathrm{tr}}}\right)$, and the computed (approximate) one, $r_{0}=\left(r_{x_{0}}, r_{y_{0}}, r_{z_{0}}\right)$, and $\widehat{\Delta r}=\hat{r}-r_{0}$ is the position vector difference between the estimated position vector $\hat{r}=\left(\hat{r}_{x}, \hat{r}_{y}, \hat{r}_{z}\right)$ and $r_{0}$.

Based on the linear system (Eq. 6) and the observation error modelled using a Gaussian distribution, i.e. $\varepsilon \sim$ $\mathscr{N}\left(0, \Gamma_{\varepsilon}\right)$, the estimated position is given by

$\hat{r}=r_{0}+\left(A^{\mathrm{T}} \Gamma_{\varepsilon}^{-1} A\right)^{-1} A^{\mathrm{T}} \Gamma_{\varepsilon}^{-1} b$.

The statistical expectation, denoted by $\mathbb{E}[\cdot]$, and covariance, denoted by $\operatorname{Cov}[\cdot]$, of $\hat{r}$ are

$$
\begin{aligned}
\mathbb{E}[\hat{r}] & =r_{0}+\left(A^{\mathrm{T}} \Gamma_{\varepsilon}^{-1} A\right)^{-1} A^{\mathrm{T}} \Gamma_{\varepsilon}^{-1} \mathbb{E}[b]=r_{\mathrm{tr}} \\
\operatorname{Cov}[\hat{r}] & =\left(A^{\mathrm{T}} \Gamma_{\varepsilon}^{-1} A\right)^{-1} A^{\mathrm{T}} \Gamma_{\varepsilon}^{-1} \operatorname{Cov}[b] \Gamma_{\varepsilon}^{-1} A\left(A^{\mathrm{T}} \Gamma_{\varepsilon}^{-1} A\right)^{-1} \\
& =\left(A^{\mathrm{T}} \Gamma_{\varepsilon}^{-1} A\right)^{-1}=\Gamma,
\end{aligned}
$$

where $\mathbb{E}[b]=\mathbb{E}\left[A\left(r_{\mathrm{tr}}-r_{0}\right)+\varepsilon\right], \mathbb{E}[\varepsilon]=0$ and $\operatorname{Cov}[b]=\Gamma_{\varepsilon}$.
The position error vector between the true and the estimation position vector is defined as $e=\left(e_{x}, e_{y}, e_{z}\right)=\hat{r}-r_{\mathrm{tr}}$. The statistical expectation and covariance of the position error are, respectively,

$$
\mathbb{E}[e]=\mathbb{E}[\hat{r}]-r_{\mathrm{tr}}=0
$$

$\operatorname{Cov}[e]=\operatorname{Cov}[\hat{r}]=\Gamma$.

The average square magnitude of the position error, given by $\|e\|^{2}=e_{x}^{2}+e_{y}^{2}+e_{z}^{2}=\left(\hat{r}_{x}-r_{x_{\mathrm{tr}}}\right)^{2}+\left(\hat{r}_{y}-r_{y_{\mathrm{tr}}}\right)^{2}+\left(\hat{r}_{z}-\right.$ $\left.r_{z_{\text {tr }}}\right)^{2}$, is

$$
\begin{aligned}
\mathbb{E}\left[\|e\|^{2}\right] & =\mathbb{E}\left[e_{x}^{2}\right]+\mathbb{E}\left[e_{y}^{2}\right]+\mathbb{E}\left[e_{z}^{2}\right] \\
& =\operatorname{tr}(\operatorname{Cov}[e])=\operatorname{tr}(\Gamma)=\operatorname{tr}\left(\left(A^{\mathrm{T}} \Gamma_{\varepsilon}^{-1} A\right)^{-1}\right)
\end{aligned}
$$

From Eq. 35, we get that

$\mathbb{E}\left[\|\mathrm{e}\|^{2}\right]=\gamma \operatorname{PDOP}_{X Y Z}^{2}$

where $\gamma$ is a scaling factor. Finally,

$\operatorname{PDOP}_{X Y Z} \propto \sqrt{\mathbb{E}\left[\|e\|^{2}\right]}$

Therefore, we can see that the position dilution of precision is proportional to the root of the expected square magnitude of the position error. ${ }^{6}$ We emphasize that the estimation of position error statistics would require real measurements taken for a fixed matrix $A$ (i.e. for a fixed satellite constellation) over a very long period of time. This kind of requirement is in most cases far from feasible (obviously because the available satellite constellations change over time) which makes the model-based PDOP so crucial in quantifying the expected position errors in positioning and navigation systems.

\footnotetext{
${ }^{6}$ In reality, the true covariance of the observation errors, denoted by $\Gamma_{\varepsilon}^{\text {true }}$, is only partly known. Of course, the better we can approximate the true covariance $\Gamma_{\varepsilon}^{\text {true }}$, the better we can predict the expected error between the true and the estimated position by using the PDOP. If the approximation $\Gamma_{\varepsilon} \approx \Gamma_{\varepsilon}^{\text {true }}$, we end up with the well-known result (Eq. 43). However, if $\Gamma_{\varepsilon} \not \Gamma_{\varepsilon}^{\text {true }}$, then Eq. 43 is not valid anymore. For example, during high-scintillation activity we aim to improve the approximation $\Gamma_{\varepsilon} \approx \Gamma_{\varepsilon}^{\text {true }}$ by introducing statistical knowledge concerning scintillation into $\Gamma_{\varepsilon}$ and thus estimate a weighted PDOP that satisfies Eq. 43.
} 


\section{References}

Aarons J (1982) Global morphology of ionospheric scintillations. Proc IEEE 70(4):360-378. https://doi.org/10.1109/PROC.1982.12314

Abadi P, Saito S, Srigutomo W (2014) Low-latitude scintillation occurrences around the equatorial anomaly crest over indonesia. Ann Geophys 32(1):7-17. https://doi.org/10.5194/angeo-32-7-2014

Alfonsi LL, Nazionale di Geofisica e Vulcanologia I, Roma SRI, De Franceschi G, Romano V (2018) Positioning errors during space weather-event of October 2003

Appleton EV (1946) Two anomalies in the ionosphere. Nature 157(3995):691-691. https://doi.org/10.1038/157691a0

Aquino M, Moore T, Dodson A, Waugh S, Souter J, Rodrigues FS (2005) Implications of ionospheric scintillation for GNSS users in northern Europe. J Navig 58(2):241-256. https://doi.org/10.1017/ S0373463305003218

Aquino M, Monico JFG, Dodson AH, Marques H, Franceschi GD, Alfonsi L, Romano V, Andreotti M (2009) Improving the GNSS positioning stochastic model in the presence of ionospheric scintillation. J Geod 83(10):953-966. https://doi.org/10.1007/s00190009-0313-6

Basu S, MacKenzie E, Basu S (1988) Ionospheric constraints on VHF/UHF communications links during solar maximum and minimum periods. Radio Sci 23(03):363-378. https://doi.org/10.1029/ RS023i003p00363

Béniguel Y, Adam JP, Bourdillon A, Lassudrie-Duchesne P (2011) Ionosphere scintillation effects on navigation systems. C R Phys 12(2):186-191. https://doi.org/10.1016/j.crhy.2011.01.005

Chen CS, Chiu YJ, Lee CT, Lin JM (2013) Calculation of weighted geometric dilution of precision. J Appl Math 2013:1-10. https:// doi.org/10.1155/2013/953048

CIGALA/CALIBRA (b.d. 2009) Monitoring stations from the network were deployed in the context of the Projects CIGALA and CALIBRA both funded by the European Commission (EC) in the framework of the FP7-GALILEO-2009-GSA and FP7-GALILEO-2011-GSA-1a, respectively, and FAPESP Project Number 06/04008-2. http://is-cigala-calibra.fct.unesp.br/is/

Conker RS, El-Arini MB, Hegarty CJ, Hsiao T (2003) Modeling the effects of ionospheric scintillation on GPS/satellite-based augmentation system availability. Radio Sci 38(1):1-1-1-23. https://doi. org/10.1029/2000rs002604

Crane RK (1977) Ionospheric scintillation. Proc IEEE 65(2):180-199. https://doi.org/10.1109/PROC.1977.10456

da Silva HA, de Oliveira Camargo P, Monico JFG, Aquino M, Marques HA, Franceschi GD, Dodson A (2010) Stochastic modelling considering ionospheric scintillation effects on GNSS relative and point positioning. Adv Space Res 45(9):1113-1121. https://doi. org/10.1016/j.asr.2009.10.009

Davies K (1990) Ionospheric radio. Institution of Engineering \& Technology, London

de Moraes AO, Vani BC, Costa E, Abdu MA, de Paula ER, Sousasantos J, Monico JFG, Forte B, de Siqueira Negreti PM, Shimabukuro MH (2018) GPS availability and positioning issues when the signal paths are aligned with ionospheric plasma bubbles. GPS Solut. https://doi.org/10.1007/s10291-018-0760-8

de Rezende LFC, de Paula ER, Kantor IJ, Kintner PM (2006) Mapping and survey of plasma bubbles over Brazilian territory. J Navig 60(01):69. https://doi.org/10.1017/s0373463307004006

Forte B, Coleman C, Skone S, Häggström I, Mitchell C, Dalt FD, Panicciari T, Kinrade J, Bust G (2017) Identification of scintillation signatures on GPS signals originating from plasma structures detected with EISCAT incoherent scatter radar along the same line of sight. J Geophys Res: Space Phys 122(1):916-931. https://doi. org/10.1002/2016ja023271
Guo K, Aquino M, Veettil SV (2019) Ionospheric scintillation intensity fading characteristics and GPS receiver tracking performance at low latitudes. GPS Solut. https://doi.org/10.1007/s10291-0190835-1

Jacobsen KS, Dähnn M (2014) Statistics of ionospheric disturbances and their correlation with GNSS positioning errors at high latitudes. J Space Weather Space Clim 4:A27. https://doi.org/10. $1051 /$ swsc/2014024

Jiao Y, Morton YT (2015) Comparison of the effect of high-latitude and equatorial ionospheric scintillation on gps signals during the maximum of solar cycle 24. Radio Sci 50(9):886-903

Jiao Y, Xu D, Morton Y, Rino C (2016) Equatorial scintillation amplitude fading characteristics across the GPS frequency bands. Navigation 63(3):267-281. https://doi.org/10.1002/navi.146

Jiao Y, Xu D, Rino CL, Morton YT, Carrano CS (2018) A multifrequency GPS signal strong equatorial ionospheric scintillation simulator: algorithm, performance, and characterization. IEEE Trans Aerosp Electron Syst 54(4):1947-1965. https://doi.org/10. $1109 /$ taes.2018.2805232

Kaipio JP, Somersalo E (2004) Statistical and computational inverse problems. Applied mathematical series. Springer, Berlin

Kintner PM, Ledvina BM (2005) The ionosphere, radio navigation, and global navigation satellite systems. Adv Space Res 35(5):788-811. https://doi.org/10.1016/j.asr.2004.12.076

Kintner PM, Ledvina BM, de Paula ER (2007) GPS and ionospheric scintillations. Space Weather. https://doi.org/10.1029/ 2006sw000260

Langley RB (1999) Dilution of precision. GPS World

Langley R (2012) How irregularities in electron density perturb satellite navigation systems. https://www.gpsworld.com/gnsssystemsignal-processinginnovation-ionospheric-scintillations12809 /

Li G, Ning B, Zhao B, Liu L, Liu J, Yumoto K (2008) Effects of geomagnetic storm on GPS ionospheric scintillations at sanya. J Atmos Solar-Terr Phys 70(7):1034-1045. https://doi.org/10.1016/j.jastp. 2008.01.003

Li G, Ning B, Ren Z, Hu L (2010) Statistics of GPS ionospheric scintillation and irregularities over polar regions at solar minimum. GPS Solut 14(4):331-341. https://doi.org/10.1007/s10291-009-0156$\mathrm{x}$

Liu K, Li G, Ning B, Hu L, Li H (2015) Statistical characteristics of low-latitude ionospheric scintillation over china. Adv Space Res 55(5):1356-1365. https://doi.org/10.1016/j.asr.2014.12.001

Luo X, Lou Y, Xiao Q, Gu S, Chen B, Liu Z (2018) Investigation of ionospheric scintillation effects on BDS precise point positioning at low-latitude regions. GPS Solut. https://doi.org/10.1007/ s10291-018-0728-8

Mainul M, Jakowski N (2012) Ionospheric propagation effects on GNSS signals and new correction approaches. In: Global navigation satellite systems: signal, theory and applications, InTech. https://doi. org/10.5772/30090

Marques HAS, Monico JFG, Marques HA (2015) Performance of the 12 c civil GPS signal under various ionospheric scintillation effects. GPS Solut 20(2):139-149. https://doi.org/10.1007/s10291-0150472-2

Marques HA, Marques HAS, Aquino M, Veettil SV, Monico JFG (2018) Accuracy assessment of precise point positioning with multiconstellation GNSS data under ionospheric scintillation effects. J Space Weather Space Clim 8:A15. https://doi.org/10.1051/swsc/ 2017043

Misra P, Enge P (2010) Global positioning system: signals, measurements, and performance (Revised Second Edition). Ganga-Jamuna Press. https://www.amazon.com/ Global-Positioning-System-Measurements-Performance/dp/ 0970954425?SubscriptionId=AKIAIOBINVZYXZQZ2U3A\& 
tag $=$ chimbori05-20\&linkCode $=x \operatorname{xm} \&$ camp $=2025 \&$ creative $=165953 \&$ creative $A S I N=0970954425$

Moreno B, Radicella S, de ML, Herraiz M, Caderot GR (2011) On the effects of the ionospheric disturbances on precise point positioning at equatorial latitudes. GPS Solut 15(4):381-390. https://eprints. ucm.es/17729/

Murphy KP (2012) Machine learning. MIT Press Ltd, Cambridge

Olatunbosun L (2017) Investigation of scintillation occurrence during intense geomagnetic storms at low latitude stations. Phys Astron Int J. https://doi.org/10.15406/paij.2017.01.00005

Oppe S (1988) The concept of risk: a decision theoretic approach. Ergonomics 31(4):435-440. https://doi.org/10.1080/ 00140138808966690

Paul A, DasGupta A (2010) Characteristics of the equatorial ionization anomaly in relation to the day-to-day variability of ionospheric irregularities around the postsunset period. Radio Sci. https://doi. org/10.1029/2009rs004329

Peng Y, Scales WA (2019) Satellite formation flight simulation using multi-constellation GNSS and applications to ionospheric remote sensing. Remote Sens 11(23):2851. https://doi.org/10. 3390/rs11232851

Pi X, Iijima BA, Lu W (2017) Effects of ionospheric scintillation on GNSS-based positioning. Navigation 64(1):3-22. https://doi.org/10.1002/navi.182. https://onlinelibrary.wiley.com/ doi/abs/10.1002/navi.182

Priyadarshi S (2015) A review of ionospheric scintillation models. Surv Geophys 36(2):295-324. https://doi.org/10.1007/s10712015-9319-1

Rufenach CL (1972) Power-law wavenumber spectrum deduced from ionospheric scintillation observations. J Geophys Res 77(25):4761-4772. https://doi.org/10.1029/ja077i025p04761

Sahithi K, Sridhar M, Kotamraju SK, Kavya KS, Sivavaraprasad G, Ratnam D, Deepthi C (2019) Characteristics of ionospheric scintillation climatology over indian low-latitude region during the 24th solar maximum period. Geod Geodyn. https://doi.org/10.1016/j. geog.2018.11.006

Sairo H, Akopian D, Takala J (2003) Weighted dilution of precision as quality measure in satellite positioning. IEE Proc Radar Sonar Navig 150(6):430-436. https://doi.org/10.1049/ip-rsn:20031008

Skone S (2001) The impact of magnetic storms on GPS receiver performance. J Geod 75(9-10):457-468

Skone S, Knudsen K, de Jong M (2001) Limitations in GPS receiver tracking performance under ionospheric scintillation conditions. Phys Chem Earth, Part A: Solid Earth Geod 26(6):613-621. https://doi.org/10.1016/S1464-1895(01)00110-7. http://www. sciencedirect.com/science/article/pii/S1464189501001107. Proceedings of the first COST action 716 workshop towards operational GPS meteorology and the second network workshop of the international GPS service (IGS)
Spogli L, Alfonsi L, Franceschi GD, Romano V, Aquino MHO, Dodson A (2009) Climatology of GPS ionospheric scintillations over high and mid-latitude european regions. Ann Geophys 27(9):3429_ 3437. https://doi.org/10.5194/angeo-27-3429-2009

Sreeja VV, Aquino M, Forte B, Elmas Z, Hancock C, Franceschi GD, Alfonsi L, Spogli L, Romano V, Bougard B, Monico JFG, Wernik AW, Sleewaegen JM, Cantó A, Silva EFD (2011) Tackling ionospheric scintillation threat to GNSS in Latin America. J Space Weather Space Clim 1(1):A05. https://doi.org/10.1051/ swsc/2011005

Tahsin M, Sultana S, Reza T, Hossam-E-Haider M (2015) Analysis of dop and its preciseness in gnss position estimation. In: 2015 International conference on electrical engineering and information communication technology (ICEEICT), pp 1-6. https://doi.org/10. 1109/ICEEICT.2015.7307445

Takahashi H, Wrasse CM, Denardini CM, Pádua MB, de Paula ER, Costa SMA, Otsuka Y, Shiokawa K, Monico JFG, Ivo A, Sant'Anna N (2016) Ionospheric TEC weather map over South America. Space Weather 14(11):937-949. https://doi.org/ 10.1002/2016sw001474

Teng Y, Wang J, Huang Q, Liu B (2018) New characteristics of weighted GDOP in multi-GNSS positioning. GPS Solut. https://doi.org/10. 1007/s10291-018-0740-z

Teunissen PJG, Kleusberg A (1998) GPS observation equations and positioning concepts. In: GPS for geodesy, Springer Berlin Heidelberg, pp 175-217. https://doi.org/10.1007/bfb0117682

van den IJssel J, Forte B, Montenbruck O (2016) Impact of swarm GPS receiver updates on POD performance. Earth Planets Space. https://doi.org/10.1186/s40623-016-0459-4

Vani BC, Shimabukuro MH, Monico JFG (2017) Visual exploration and analysis of ionospheric scintillation monitoring data: the ISMR query tool. Comput Geosci 104:125-134. https://doi.org/10.1016/ j.cageo.2016.08.022

Wernik A, Secan J, Fremouw E (2003) Ionospheric irregularities and scintillation. Adv Space Res 31(4):971-981. https://doi.org/10. 1016/s0273-1177(02)00795-0

Xu R, Liu Z, Li M, Morton Y, Chen W (2012) An analysis of lowlatitude ionospheric scintillation and its effects on precise point positioning. J Glob Position Syst 11(1):22-32. https://doi.org/10. 5081/jgps.11.1.22

Zhang X, Guo F, Zhou P (2013) Improved precise point positioning in the presence of ionospheric scintillation. GPS Solut 18(1):51-60. https://doi.org/10.1007/s10291-012-0309-1

Zumberge JF, Heflin MB, Jefferson DC, Watkins MM, Webb FH (1997) Precise point positioning for the efficient and robust analysis of GPS data from large networks. J Geophys Res: Solid Earth 102(B3):5005-5017. https://doi.org/10.1029/96jb03860 